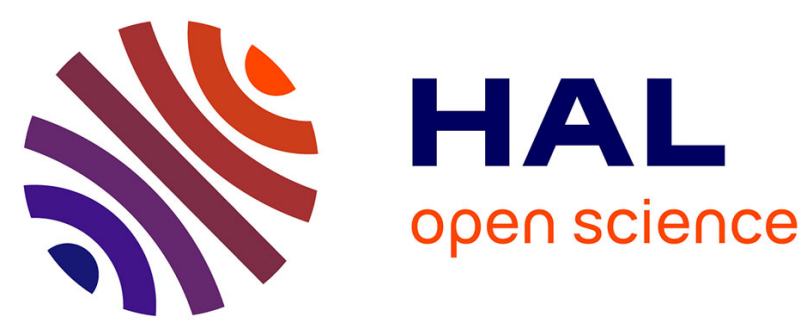

\title{
Optimal control of a coupled partial and ordinary differential equations system for the assimilation of polarimetry Stokes vector measurements in tokamak free-boundary equilibrium reconstruction with application to ITER
}

Blaise Faugeras, Jacques Blum, Holger Heumann, Cedric Boulbe

\section{To cite this version:}

Blaise Faugeras, Jacques Blum, Holger Heumann, Cedric Boulbe. Optimal control of a coupled partial and ordinary differential equations system for the assimilation of polarimetry Stokes vector measurements in tokamak free-boundary equilibrium reconstruction with application to ITER. Computer Physics Communications, 2017, 217, pp.43 - 57. 10.1016/j.cpc.2017.04.003 . hal-01442462v2

HAL Id: hal-01442462

https://hal.inria.fr/hal-01442462v2

Submitted on 5 Dec 2017

HAL is a multi-disciplinary open access archive for the deposit and dissemination of scientific research documents, whether they are published or not. The documents may come from teaching and research institutions in France or abroad, or from public or private research centers.
L'archive ouverte pluridisciplinaire HAL, est destinée au dépôt et à la diffusion de documents scientifiques de niveau recherche, publiés ou non, émanant des établissements d'enseignement et de recherche français ou étrangers, des laboratoires publics ou privés. 


\title{
Optimal control of a coupled partial and ordinary differential equations system for the assimilation of polarimetry Stokes vector measurements in tokamak free-boundary equilibrium reconstruction with application to ITER
}

\author{
Blaise Faugeras $^{\mathrm{a}, *}$, Jacques Blum$^{\mathrm{a}}$, Holger Heumann ${ }^{\mathrm{a}}$, Cédric Boulbe ${ }^{\mathrm{a}}$ \\ ${ }^{a}$ Université Côte d'Azur, CNRS, Inria, LJAD, Parc Valrose, 06108 Nice Cedex 2, France
}

\begin{abstract}
The modelization of polarimetry Faraday rotation measurements commonly used in tokamak plasma equilibrium reconstruction codes is an approximation to the Stokes model. This approximation is not valid for the foreseen ITER (International Thermonuclear Experimental Reactor) scenarios where high current and electron density plasma regimes are expected. In this work a method enabling the consistent resolution of the inverse equilibrium reconstruction problem in the framework of non-linear free-boundary equilibrium coupled to the Stokes model equation for polarimetry is provided. Using optimal control theory we derive the optimality system for this inverse problem. A sequential quadratic programming (SQP) method is proposed for its numerical resolution. Numerical experiments with noisy synthetic measurements in the ITER tokamak configuration for two test cases, the second of which is an H-mode plasma, show that the method is efficient and that the accuracy of the identification of the unknown profile functions is improved compared to the use of classical Faraday measurements.

Keywords: Tokamak equilibrium reconstruction, free plasma boundary, Stokes model polarimetry, inverse problem, PDE-constrained optimization
\end{abstract}

\footnotetext{
* Corresponding author

Email address: blaise.faugeras@unice.fr (Blaise Faugeras )
}

Preprint submitted to Computer Physics Communications

December 5, 2017 
PACS: 02.03.Zz, 02.60.-x, 52.55.-s, 52.55.Fa, 52.65.-y

\section{Introduction}

Numerical equilibrium reconstruction is an important and long standing subject in tokamak fusion plasma science [1, 2, 3, 4, 5, 6, The resolution of this inverse problem: the reconstruction of the poloidal flux function and of the plasma boundary as well as the identification of two non-linear source term functions known as $p^{\prime}$ and $f f^{\prime}$ in the Grad-Shafranov equation [7, 8, 9] (see Eq. (6) below), is needed on the one hand for real time control of the plasma during a discharge and on the other hand for post-treatment analysis of equilibrium configurations. The basic set of measurements needed and used are magnetic probes and flux loops which provide values of the poloidal magnetic field and flux at several points surrounding the vacuum vessel and the plasma. All free boundary reconstruction codes (e.g. [10, 11, 12, 13, 14, 15, 16, 17]) primarily use these magnetic measurements which proved to be sufficient to identify correctly the plasma boundary and the averaged plasma current density profile [15. However the difficulty of the reconstruction of the current profile, when only magnetic measurements are used, has been pointed out in [18] and is inherent to the ill-posedness of this inverse problem.

In order to be able to separate more precisely the contributions of the two non-linearities $p^{\prime}$ and $f f^{\prime}$ it appears necessary to use supplementary measurements of interferometry and polarimetry which provide integrated quantities along lines of sight or chords crossing the plasma poloidal section [19, 10, 11, 20, 15. Concerning polarimetry, equilibrium reconstruction codes have until now use Faraday rotation angle measurements only. Moreover the modelization used to represent these Faraday rotation measurements is known to be a too rough approximation for ITER plasma regimes where the Cotton-Mouton effects is expected to be much stronger than in today's tokamaks as a result of high plasma currents and high electron density. The relativistic effect is also expected to be stronger as a result of the high electron temperature [21]. 
In recent papers [22, 23, 24] it has been shown using JET measurements that for high plasma currents $I_{p}$ (in $M A$ ) and electron density $N_{e}$ (in units of $10^{20} \mathrm{~m}^{-3}$ ) the polarimetry Faraday effect cannot be considered alone independently from the Cotton-Mouton effect and that the coupling between them has to be taken into account. An approximate rule is derived analytically in [23] to determine the plasma parameters where the nonlinear effects are significant: $N_{e} \times I_{p} \geq 2.38$. In future ITER scenarios 25 , 26] the foreseen plasma current is $I_{p}=15 \mathrm{MA}$ and applying this formula one finds $N_{e} \geq 0.1610^{20} \mathrm{~m}^{-3}$ which is in the low range of density values expected in ITER scenarios. Hence for such plasma the Stokes model for polarimety [27] has to be used instead of its approximation commonly used in equilibrium reconstruction codes.

This seriously complicates the task of equilibrium reconstruction since it introduces an additional system of three ordinary differential equations, the Stokes model, along each of the polarimetry chords and additional constraints on the poloidal flux. A first step in this direction is proposed in [28, 29] on simplified plasma models. In the present work we provide for the first time a numerical method enabling the consistent resolution of the inverse equilibrium reconstruction problem in the framework of non-linear free-boundary equilibrium coupled to the Stokes model equation. The inverse problem is formulated as a non-linear least-square minimization problem under the non-linear constraints of both the equilibrium equation and the Stokes equation. This naturally encourages us to propose the use of a sequential quadratic programming (SQP) method 30].

Next Section 2 is devoted to the formulation of the direct model and the inverse problem. In Section 3 we discuss the numerical methods which we have developed for their resolution and finally in Section 4 some numerical experiments are presented for an ITER configuration on two different test cases. 


\section{Inverse problem formulation}

\subsection{Free-boundary plasma equilibrium}

The equations which govern the equilibrium of a plasma in the presence of a magnetic field in a tokamak are on the one hand Maxwell's equations satisfied in the whole of space (including the plasma):

$$
\nabla \cdot \mathbf{B}=0, \nabla \times\left(\frac{\mathbf{B}}{\mu}\right)=\mathbf{j}
$$

and on the other hand the equilibrium equation for the plasma itself

$$
\nabla p=\mathbf{j} \times \mathbf{B},
$$

where $\mathbf{B}$ is the magnetic field, $\mu$ is the magnetic permeability, $p$ is the kinetic pressure and $\mathbf{j}$ is the current density. We refer to standard text books (e.g. [31, 6, 32, 33, 34]) and to [35. for details of the derivation and only state the needed equations in what follows.

Introducing a cylindrical coordinate system $\left(\boldsymbol{e}_{r}, \boldsymbol{e}_{\phi}, \boldsymbol{e}_{z}\right)(r=0$ is the major axis of the tokamak torus) and assuming axial symmetry equations (1) and (2) reduce to the following equation for the poloidal flux $\psi(r, z)$ in the poloidal plane $\Omega_{\infty}=(0, \infty) \times(-\infty, \infty)$ :

$$
-\Delta^{*} \psi=j_{\phi}
$$

where $j_{\phi}$ is the toroidal component of $\mathbf{j}$, and the second order elliptic differential operator $\Delta^{*}$ is defined by

$$
\Delta^{*} .:=\partial_{r}\left(\frac{1}{\mu_{0} r} \partial_{r} \cdot\right)+\partial_{z}\left(\frac{1}{\mu_{0} r} \partial_{z} \cdot\right):=\nabla \cdot\left(\frac{1}{\mu_{0} r} \nabla .\right) .
$$

Here $\nabla$ is the $2 \mathrm{D}$ operator in the $(r, z)$-plane and $\mu_{0}$ is the magnetic permeability of vacuum (in this work we consider only air-transformer tokamaks such as ITER).

The magnetic field can be decomposed in poloidal and toroidal components

$$
\mathbf{B}=\mathbf{B}_{p}+\mathbf{B}_{\phi}, \quad \mathbf{B}_{p}=\frac{1}{r}\left[\nabla \psi \times \boldsymbol{e}_{\phi}\right], \quad \boldsymbol{B}_{\phi}=B_{\phi} \mathbf{e}_{\phi}=\frac{f}{r} \boldsymbol{e}_{\phi},
$$



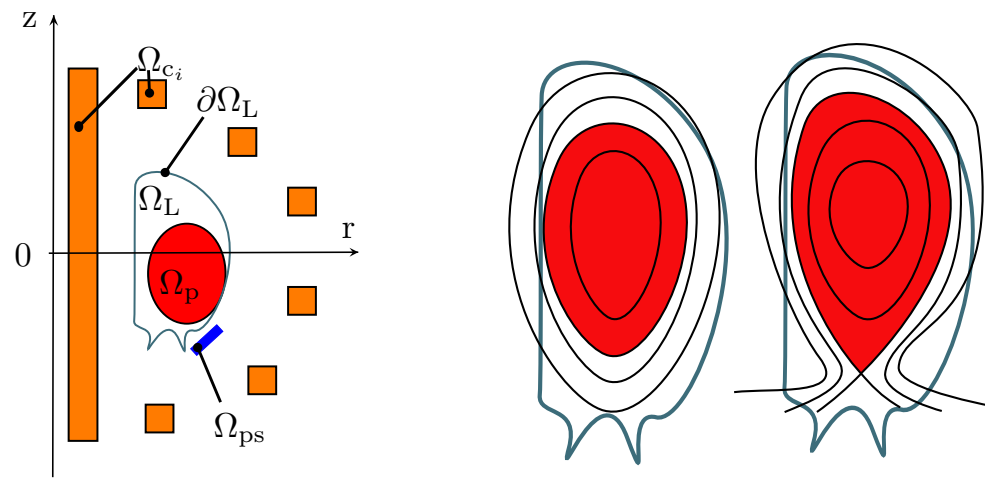

Figure 1: Left: schematic representation of the poloidal plane of a tokamak. $\Omega_{\mathrm{p}}$ is the plasma domain, $\Omega_{\mathrm{L}}$ is the limiter domain accessible to the plasma, $\Omega_{\mathrm{c}_{i}}$ represent poloidal field coils, $\Omega_{\mathrm{ps}}$ the passive structures. Right: example of a plasma whose boundary is defined by the contact with limiter (left) or by the presence of an X-point (right).

where $f$ is the diamagnetic function. Equation (5) shows that the magnetic surfaces are generated by the rotation of the iso-flux lines around the axis of the torus.

The toroidal component of the current density $j_{\phi}$ is zero everywhere outside the plasma domain and the poloidal field coils (and possibly the passive structures). The different sub-domains of the poloidal plane of a schematic tokamak (see Fig. 1) as well as the corresponding expression for $j_{\phi}$ are described below:

$-\Omega_{\mathrm{L}}$ is the domain accessible to the plasma. Its boundary is the limiter $\partial \Omega_{\mathrm{L}}$.

$-\Omega_{\mathrm{p}}$ is the plasma domain where equations (2) and (1) imply that $p$ and $f$ are constant on each magnetic surface i.e. $p=p(\psi)$ and $f=f(\psi)$. One then deduces the so-called Grad-Shafranov equilibrium equation in the plasma [7, 8, 9]

$$
-\Delta^{*} \psi=r p^{\prime}(\psi)+\frac{1}{\mu_{0} r}\left(f f^{\prime}\right)(\psi) .
$$

The right-hand side of (6) is the toroidal component $j_{\phi}$ of the plasma current density.

The plasma domain is unknown, i.e. $\Omega_{\mathrm{p}}=\Omega_{\mathrm{p}}(\psi)$, and this is a free boundary problem. This domain is defined by its boundary which is the outermost closed 
$\psi$ iso-contour contained within the limiter $\Omega_{\mathrm{L}}$. The plasma can either be limited if this iso-contour is tangent to the limiter $\partial \Omega_{\mathrm{L}}$ or defined by the presence of an $\mathrm{X}$-point (see Fig. 1). In the latter case the plasma domain is strictly bounded by the magnetic separatrix.

More precisely

$$
\begin{gathered}
\Omega_{\mathrm{p}}(\psi)=\left\{(r, z) \in \Omega_{\mathrm{L}},\right. \\
\left.\psi(r, z) \geq \max \left(\max _{(r, z) \in \partial \Omega_{\mathrm{L}}} \psi(r, z), \max _{\left(r_{X}, z_{X}\right) \in \Omega_{\mathrm{L}}} \psi\left(r_{\mathrm{X}}, z_{\mathrm{X}}\right)\right)\right\},
\end{gathered}
$$

where $\left(r_{\mathrm{X}}, z_{\mathrm{X}}\right)$ denotes the coordinates of the saddle points of $\psi$. The functions $p^{\prime}$ and $f f^{\prime}$ are zero outside $\Omega_{\mathrm{p}}$.

The current density is non-linear in $\psi$ due to the non-linear functions $p^{\prime}$ and $f f^{\prime}$ and the definition of the plasma domain $\Omega_{\mathrm{p}}(\psi)$. While $\Omega_{\mathrm{p}}(\psi)$ is fully determined for a given $\psi$, the two functions $p^{\prime}$ and $f f^{\prime}$ are not determined in this modelization. It is the goal of the inverse equilibrium reconstruction problem to determine them. For now let us consider that we are given two functions $A\left(\psi_{\mathrm{N}}\right)$ and $B\left(\psi_{\mathrm{N}}\right)$ such that

$$
j_{\phi}=\lambda\left(\frac{r}{r_{0}} A\left(\psi_{\mathrm{N}}\right)+\frac{r_{0}}{r} B\left(\psi_{\mathrm{N}}\right)\right) .
$$

Here $r_{0}$ is the major radius of the tokamak vacuum chamber and $\lambda$ is a scaling coefficient. The normalized poloidal flux $\psi_{\mathrm{N}}(r, z)$ is

$$
\psi_{\mathrm{N}}(r, z)=\frac{\psi(r, z)-\psi_{\mathrm{a}}(\psi)}{\psi_{\mathrm{b}}(\psi)-\psi_{\mathrm{a}}(\psi)} .
$$

with $\psi_{\mathrm{a}}$ and $\psi_{\mathrm{b}}$ being the flux values at the magnetic axis and at the boundary of the plasma:

$$
\begin{aligned}
& \psi_{\mathrm{a}}(\psi):=\psi\left(r_{\mathrm{a}}(\psi), z_{\mathrm{a}}(\psi)\right), \\
& \psi_{\mathrm{b}}(\psi):=\psi\left(r_{\mathrm{b}}(\psi), z_{\mathrm{b}}(\psi)\right),
\end{aligned}
$$

with $\left(r_{\mathrm{a}}(\psi), z_{\mathrm{a}}(\psi)\right)$ the magnetic axis, where $\psi$ has its global maximum in $\Omega_{\mathrm{L}}$ and $\left(r_{\mathrm{b}}(\psi), z_{\mathrm{b}}(\psi)\right)$ the coordinates of the point that determines the plasma boundary. The point $\left(r_{\mathrm{b}}, z_{\mathrm{b}}\right)$ is either an X-point of $\psi$ or the contact point with the limiter $\partial \Omega_{\mathrm{L}}$. 
-Domains $\Omega_{\mathrm{c}_{i}}$ represent the poloidal field coils carrying currents.

$$
j_{\phi}=\frac{I_{i}}{S_{i}},
$$

where $S_{i}$ is the section area of the coil and $I_{i}$ is a given measured current. $\Omega_{\mathrm{ps}}$ represents passive structures where the induced current density is assumed to be 0 in this work but can be considered to be measured and given in the same form as Eq. 10

To sum up, given functions $A$ and $B$, and currents $\mathbf{I}=\left\{I_{i}\right\}_{i=1}^{N_{C}}$ in the coils, the free-boundary equilibrium equation for $\psi(r, z)$ on $\Omega_{\infty}$ is the following nonlinear boundary value problem

$$
\begin{cases}-\Delta^{*} \psi= \begin{cases}\lambda\left(\frac{r}{r_{0}} A\left(\psi_{\mathrm{N}}\right)+\frac{r_{0}}{r} B\left(\psi_{\mathrm{N}}\right)\right) & \text { in } \Omega_{\mathrm{p}}(\psi), \\ \frac{I_{i}}{S_{i}} & \text { in } \Omega_{\mathrm{c}_{i}}, \\ 0 & \text { elsewhere }\end{cases} \\ \psi(0, z, t)=0, & \\ \lim _{\|(r, z)\| \rightarrow+\infty} \psi(r, z)=0 . & \end{cases}
$$

This formulation on an infinite domain is not used directly in computations where we use finite elements on a truncated bounded domain. The infinite domain is reduced to a semi circular computational domain by an uncoupling procedure [36, 37]. We chose a semi-circle $\Gamma$ of radius $\rho_{\Gamma}$ surrounding the coil domains $\Omega_{\mathrm{c}_{i}}$ (see Fig. 2 and define the computation domain $\Omega$ having boundary $\partial \Omega=\Gamma \cup \Gamma_{0}$, where $\Gamma_{0}=\left\{(0, z), z \in\left[-\rho_{\Gamma}, \rho_{\Gamma}\right]\right\}$. The weak formulation of the equilibrium problem on which the finite element method relies uses the following function space

$$
V:=\left\{\psi: \Omega \rightarrow \mathbb{R},\|\psi\|<\infty,\left\|\frac{|\nabla \psi|}{r}\right\|<\infty, \psi_{\mid \Gamma_{0}}=0\right\} \cap C^{0}(\bar{\Omega}),
$$

with

$$
\|\psi\|^{2}=\int_{\Omega} \psi^{2} r d r d z
$$

and can be written as:

Given function $A$ and $B$, and currents $\mathbf{I}$, find $\psi \in V$ such that for all $\xi \in V$

$$
\mathrm{a}(\psi, \xi)-\mathrm{J}_{\mathrm{p}}(\psi, \xi ; A, B)+\mathrm{c}(\psi, \xi)=\ell(\mathbf{I}, \xi)
$$


where

$$
\begin{aligned}
\mathrm{a}(\psi, \xi) & :=\int_{\Omega} \frac{1}{\mu_{0} r} \nabla \psi \cdot \nabla \xi d r d z \\
\mathrm{~J}_{\mathrm{p}}(\psi, \xi ; A, B) & :=\int_{\Omega_{\mathrm{p}}(\psi)} \lambda\left(\frac{r}{r_{0}} A\left(\psi_{\mathrm{N}}\right)+\frac{r_{0}}{r} B\left(\psi_{\mathrm{N}}\right)\right) \xi d r d z, \\
\ell(\mathbf{I}, \xi) & :=\sum_{i=1}^{N_{C}} \frac{I_{i}}{S_{i}} \int_{\Omega_{\mathrm{c}_{i}}} \xi d r d z,
\end{aligned}
$$

and the bilinear form $\mathrm{c}: V \times V \rightarrow \mathbb{R}$ is accounting for the boundary conditions at infinity. We refer to [35] for its precise expression and to [38, Chapter 2.4] for the details on its the derivation.

\subsection{Stokes model for polarimetry}

Polarimetry consists in measurements of the change of state of polarization of an electromagnetic radiation propagating across the magnetized plasma along several chords distributed on the poloidal section of the tokamak. One method of describing the state of polarization is to introduce a Stokes vector $\mathbf{s}=\left(s_{1}, s_{2}, s_{3}\right)$. The evolution of the polarization when the laser beam crosses the plasma is then given by the following Stokes equation on each chord:

$$
\left\{\begin{array}{l}
\frac{d \mathbf{s}}{d Z}=\mathbf{G} \mathbf{s}, \quad \text { on }\left(Z_{0}, Z_{1}\right], \\
\mathbf{s}\left(Z_{0}\right)=\mathbf{s}_{0} .
\end{array}\right.
$$

We refer to [27] for details on this modelization. Here we have introduced a coordinate system $\left(\boldsymbol{e}_{X}, \boldsymbol{e}_{Y}, \boldsymbol{e}_{Z}\right)$ attached to a chord $C . Z$ is the coordinate tangent to the chord, $X$ represents the toroidal direction and $Y$ the direction perpendicular to $Z$ in the poloidal plane. In this coordinate system the components of the magnetic field are denoted by $\left(B_{X}, B_{Y}, B_{Z}\right)$.

The initial polarization is given by $\mathbf{s}_{0}$ at $Z_{0} . Z_{1}$ corresponds to the location of the output measurement sensor. The $3 \times 3$ matrix $\boldsymbol{G}$ is such that $\boldsymbol{G} \mathbf{s}=\boldsymbol{\Omega} \times \mathbf{s}$ where vector $\boldsymbol{\Omega}=\left(\Omega_{1}, \Omega_{2}, \Omega_{3}\right)$ has components

$$
\Omega_{1}=C_{1} N_{e}\left(B_{X}^{2}-B_{Y}^{2}\right), \quad \Omega_{2}=2 C_{1} N_{e} B_{X} B_{Y}, \quad \Omega_{3}=C_{3} N_{e} B_{Z}
$$


Here the electron density in the plasma, $N_{e}=N_{e}\left(\psi_{\mathrm{N}}\right)$, is assumed to be constant on the flux surfaces. The components of the magnetic field can be written as

$$
B_{Z}=\mathbf{B}_{p} \cdot \mathbf{e}_{Z}=-\frac{1}{r} \nabla \psi \cdot e_{Y}, \quad B_{Y}=\mathbf{B}_{p} \cdot \mathbf{e}_{Y}=\frac{1}{r} \nabla \psi \cdot e_{Z}, \quad B_{X}=B_{\phi}=\frac{f}{r}
$$

where the diamagnetic function $f$ is related to function $B$ through the relation $f f^{\prime}=\lambda \mu_{0} r_{0} B$. The dependence of $\boldsymbol{G}$ on $\psi$, on the density $N_{e}$ and on function $B$ is denoted by $\boldsymbol{G}\left(\psi, B, N_{e}\right)$. Constants $C_{1}$ and $C_{3}$ depend on the wavelength of the beam radiation.

Hence in order to use polarimetry measurements with Stokes modelization in addition to basic magnetic measurements for the identification of functions $A$ and $B$ one has to supplement equation 111 or 12 with, for each line of sight, a system of linear ordinary differential equations (14) for the Stokes vector.

\subsection{The inverse identification problem}

Magnetics constitute the basic set of experimental measurements used in equilibrium reconstruction for the identification of functions $A$ and $B$. They consist in measurements of projections of the poloidal magnetic field, $\mathbf{B}_{p} \cdot \mathbf{d}$ at several locations around the vacuum vessel of the tokamak (the unit vector $\mathbf{d}$ varies with each B-probe) and of measurements of $\psi$ obtained from flux loops at several locations too (see Fig. 3].

In order to be able to use polarimetric measurements the electron density function, $N_{e}\left(\psi_{\mathrm{N}}\right)$ has to be known. It is therefore also going to be identified

using interferometric measurements which give the density line integrals over each of the $N_{L}$ chords $C^{i}, i=1, \ldots N_{L}$ :

$$
N_{e, o b s}^{i} \approx \int_{C^{i}} N_{e}\left(\psi_{\mathrm{N}}\right) d Z^{i}
$$

Polarimetric measurements as they are considered in all former equilibrium reconstruction (e.g. [19, 20, 15]) studies give the Faraday rotation of the angle of the infrared radiation crossing the section of the plasma along the different chords

$$
\alpha_{o b s}^{i} \approx \frac{1}{2} \int_{C^{i}} \Omega_{3} d Z^{i}
$$


As detailed in [27] this is an approximation to one component of the Stokes vector $\mathbf{s}\left(Z_{1}^{i}\right)$ only valid for small Faraday and Cotton-Mouton effects. On the contrary in this study we consider that polarimetric measurements are given by the full Stokes vector at the $Z_{1}^{i}$ coordinate on each chord $C^{i}$

$$
\mathbf{s}_{o b s}^{i} \approx \mathbf{s}\left(Z_{1}^{i}\right)
$$

Indeed it is stated in [24, Section 2] and [27, Section 8] that the components of the Stokes vector are related directly to quantities measured by the polarimetric system.

At this point we have defined a direct model given by the equilibrium equation 12 and Stokes equation (14) on every chord, control variables $A, B$ and $N_{e}$, and measurements to which are attached experimental errors represented by the standard deviations $\sigma$ s in Eq. 19 below. The identification problem can now be formulated as a constrained minimization problem for the following cost function $\left(\{\mathbf{s}\}\right.$ denotes the vector $\left(\mathbf{s}^{1}, \ldots, \mathbf{s}^{N_{L}}\right)$ of Stokes vectors for all chords):

$$
J\left(\psi,\{\mathbf{s}\}, A, B, N_{e}\right):=J_{o b s}\left(\psi,\{\mathbf{s}\}, N_{e}\right)+R\left(A, B, N_{e}\right)
$$

where the least-square term is

$$
\begin{aligned}
& J_{o b s}\left(\psi,\{\mathbf{s}\}, N_{e}\right):= \\
& \sum_{i=1}^{N_{B}} \frac{1}{2 \sigma_{B i}^{2}}\left(\left(\mathbf{B}_{p}\left(r_{i}, z_{i}\right) \cdot \mathbf{d}_{i}\right)-B_{p, o b s}^{i}\right)^{2}+\sum_{i=1}^{N_{F}} \frac{1}{2 \sigma_{F i}^{2}}\left(\psi\left(r_{i}, z_{i}\right)-\psi_{o b s}^{i}\right)^{2} \\
& +\sum_{i=1}^{N_{L}} \frac{1}{2 \sigma_{N i}^{2}}\left(\int_{C_{i}} N_{e}\left(\psi_{\mathrm{N}}\right) d Z-N_{e, o b s}^{i}\right)^{2}+\sum_{i=1}^{N_{L}} \frac{1}{2 \sigma_{S i}^{2}}\left\|\mathbf{s}^{i}\left(Z_{1}^{i}\right)-\mathbf{s}_{o b s}^{i}\right\|^{2}
\end{aligned}
$$

and the regularization term is

$$
\begin{aligned}
& R\left(A, B, N_{e}\right)= \\
& \frac{\varepsilon_{A}}{2} \int_{0}^{1}\left[A^{\prime \prime}(x)\right]^{2} d x+\frac{\varepsilon_{B}}{2} \int_{0}^{1}\left[B^{\prime \prime}(x)\right]^{2} d x+\frac{\varepsilon_{N_{e}}}{2} \int_{0}^{1}\left[N_{e}^{\prime \prime}(x)\right]^{2} d x,
\end{aligned}
$$

under the constraint of the model equations (21) and (22) written again below:

$$
\mathrm{a}(\psi, \xi)-\mathrm{J}_{\mathrm{p}}(\psi, \xi ; A, B)+\mathrm{c}(\psi, \xi)=\ell(\mathbf{I}, \xi), \quad \forall \xi \in V,
$$


and for all chords $C^{i}, i=1, \ldots N_{L}$ :

$$
\left\{\begin{array}{l}
\frac{d \mathbf{s}^{i}}{d Z^{i}}=\boldsymbol{G}\left(\psi, B, N_{e}\right) \mathbf{s}^{i}, \quad \text { on }\left(Z_{0}^{i}, Z_{1}^{i}\right], \\
\mathbf{s}^{i}\left(Z_{0}^{i}\right)=\mathbf{s}_{0}^{i}
\end{array}\right.
$$

The unknown functions $A, B$ and $N_{e}$ are supposed to belong to a set $\mathcal{U}$ of regular functions defined on $[0,1]$.

Cost function (18) is not quadratic and the constraints are non-linear. In order to formulate the first order optimality conditions we introduce Lagrange multipliers $p$ and $\{\mathbf{q}\}$ associated respectively to constraint $(21)$ and $(22)$, and formulate the Lagrangian for the optimization problem:

$$
\begin{gathered}
\mathcal{L}\left(\psi,\{\mathbf{s}\}, A, B, N_{e}, p,\{\mathbf{q}\}\right)=J\left(\psi,\{\mathbf{s}\}, A, B, N_{e}\right) \\
+\mathrm{a}(\psi, p)-\mathrm{J}_{\mathrm{p}}(\psi, p ; A, B)+\mathrm{c}(\psi, p)-\ell(\mathbf{I}, p) \\
+\sum_{i=1}^{N_{L}} \int_{Z_{0}^{i}}^{Z_{1}^{i}}\left(\left(\frac{d \mathbf{s}^{i}}{d Z^{i}}-\boldsymbol{G}\left(\psi, B, N_{e}\right) \mathbf{s}^{i}\right) \cdot \mathbf{q}^{i}\right) d Z^{i} .
\end{gathered}
$$

A solution to the optimization problem is a stationary point of this Lagrangian. Let us formally derive the optimality system. Setting to zero the $\psi$ derivative of $\mathcal{L}$ in direction $h_{\psi}$ yields the weak formulation of adjoint equilibrium problem for $p$ :

$$
\begin{gathered}
\mathrm{a}\left(h_{\psi}, p\right)-D_{\psi} \mathrm{J}_{\mathrm{p}}(\psi, p ; A, B)\left(h_{\psi}\right)+\mathrm{c}\left(h_{\psi}, p\right)=-D_{\psi} J_{o b s}\left(\psi,\{\mathbf{s}\}, N_{e}\right)\left(h_{\psi}\right) \\
+\sum_{i=1}^{N_{L}} \int_{Z_{0}^{i}}^{Z_{1}^{i}}\left(\left(D_{\psi}\left[\boldsymbol{G}\left(\psi, B, N_{e}\right) \mathbf{s}^{i}\right]\left(h_{\psi}\right)\right) \cdot \mathbf{q}^{i}\right) d Z^{i}, \quad \forall h_{\psi} \in V .
\end{gathered}
$$

This equation involves a non-usual second term on the right hand side coming from the coupling between (21) and (22). The computation of the $\psi$ derivative of $J_{p}$ is not straightforward because of the dependence of the integration domain $\Omega_{\mathrm{p}}$ on $\psi$. We refer to [6, 35] for this point.

Setting the $\mathbf{s}^{i}$ derivative of $\mathcal{L}$ to zero yields the adjoint Stokes equations for $\mathbf{q}^{i}$ on each chord. They have to be integrated backwards from a final condition at $Z_{1}^{i}$ :

$$
\left\{\begin{array}{c}
-\frac{d \mathbf{q}^{i}}{d Z^{i}}-\boldsymbol{G}(\psi, B, n)^{T} \mathbf{q}^{i}=0, \\
\mathbf{q}^{i}\left(Z_{1}^{i}\right)=-\frac{1}{\sigma_{S_{i}}^{2}}\left(\mathbf{s}^{i}\left(Z_{1}^{i}\right)-\mathbf{s}_{o b s}^{i}\right) .
\end{array}\right.
$$


The $A, B$ and $N_{e}$ derivatives of $\mathcal{L}$ yield the gradient of cost function 18 , the constraint equations being satisfied:

$$
\begin{aligned}
& \varepsilon_{A} \int_{0}^{1} A^{\prime \prime}(x) h_{A}^{\prime \prime}(x) d x-\int_{\Omega_{\mathrm{p}}(\psi)} \lambda \frac{r}{r_{0}} h_{A}\left(\psi_{\mathrm{N}}\right) p d r d z=0, \quad \forall h_{A} \in \mathcal{U}, \\
& \varepsilon_{B} \int_{0}^{1} B^{\prime \prime}(x) h_{B}^{\prime \prime}(x) d x-\int_{\Omega_{\mathrm{p}}(\psi)} \lambda \frac{r_{0}}{r} h_{B}\left(\psi_{\mathrm{N}}\right) p d r d z \\
& -\sum_{i=1}^{N_{L}} \int_{Z_{0}^{i}}^{Z_{1}^{i}}\left(\left(D_{B}\left[\boldsymbol{G}\left(\psi, B, N_{e}\right) \mathbf{s}^{i}\right]\left(h_{B}\right)\right) \cdot \mathbf{q}^{i}\right) d Z^{i}=0, \quad \forall h_{B} \in \mathcal{U}, \\
& \varepsilon_{N_{e}} \int_{0}^{1} N_{e}^{\prime \prime}(x) h_{N_{e}}^{\prime \prime}(x) d x+D_{N_{e}} J_{o b s}\left(\psi,\{\mathbf{s}\}, N_{e}\right)\left(h_{N_{e}}\right) \\
& -\sum_{i=1}^{N_{L}} \int_{Z_{0}^{i}}^{Z_{1}^{i}}\left(\left(D_{N_{e}}\left[\boldsymbol{G}\left(\psi, B, N_{e}\right) \mathbf{s}^{i}\right]\left(h_{N_{e}}\right)\right) \cdot \mathbf{q}^{i}\right) d Z^{i}=0, \quad \forall h_{N_{e}} \in \mathcal{U} .
\end{aligned}
$$

As usual the derivative of $\mathcal{L}$ with respect to $p$ and $\mathbf{q}^{i}$ gives back the model equations (21) and 22 respectively. Equations $(24)-(25),(26)-(28)$ and 21$)-(22)$ form the optimality system for the optimization problem under consideration. In the next section we discretize it and propose a numerical algorithm for its resolution.

\section{Numerical methods}

\subsection{Discretization of the direct model}

Equilibrium equation (21) is discretized using a P1 finite element method based on triangular meshes [39, 36, 35. From now on let us also assume that functions $A$ and $B$ are decomposed in a basis of functions $\phi_{i}$ defined on $[0,1]$. We use cubic spline functions in this work and

$$
A(x)=\sum_{i=1}^{N} u_{A i} \phi_{i}(x), \quad B(x)=\sum_{i=1}^{N} u_{B i} \phi_{i}(x),
$$

and let us denote $\mathbf{u}=\left(\mathbf{u}_{A}, \mathbf{u}_{B}\right)$ the vector of degrees of freedom of $A$ and $B$

in the decomposition basis. Classically approximating $\psi$ by $\psi_{h}=\sum_{i=1}^{N_{\psi}} \psi_{i} \lambda_{i}(r, z)$ on the finite element approximation space as well as the operators of (21) and 
taking all basis elements $\lambda_{i}$ as test functions leads to the following non-linear system of $N_{\psi}$ equations:

$$
(\boldsymbol{A}+\boldsymbol{C}) \boldsymbol{\psi}-\mathbf{J}_{p}(\boldsymbol{\psi}, \mathbf{u})-\boldsymbol{L I}=0
$$

where $\boldsymbol{\psi}$ denotes the vector of finite element coefficients $\left\{\psi_{i}\right\}_{i=1}^{N_{\psi}}$ and other notations are obvious.

In order to solve direct equilibrium problem (30), $\mathbf{u}$ and $\mathbf{I}$ being given, Newton's method is used. As for the continuous case the computation of the derivative $D_{\boldsymbol{\psi}} \mathbf{J}_{p}(\boldsymbol{\psi}, \mathbf{u})\left(h_{\psi}\right)$ has to be conducted with care. In this work we use the derivatives of the discrete operator and refer to [35, section 3.2] and [35, section 3.3] for technical details.

Let us now turn to the discretization of the Stokes model for polarimetry. We use a Crank-Nicolson scheme for the integration of 22 . Each chord $C^{i}$ is discretized with $N^{i}$ points $\mathbf{x}^{i, k+1}=\mathbf{x}^{i, k}+h^{i, k} \mathbf{t}^{i}$ where $k=1 \ldots N^{i}-1, \mathbf{x}=(r, z)$ and $\mathbf{t}^{i}$ is a unit vector tangent to the chord. The first point $\mathbf{x}^{i, 1}$ corresponds to $\left(0,0, Z_{0}^{i}\right)$ in the coordinate system attached to chord $i$ and the last one $\mathbf{x}^{i, N^{i}}$ to $\left(0,0, Z_{1}^{i}\right)$. Although the discretization steps $h^{i, k}$ are chosen of the same order of magnitude as $h$, the discretization parameter for the finite element method, the $x^{i, k}$ points are independent of the triangular mesh. Let us assume that, similarly to functions $A$ and $B$, the density $N_{e}$ is decomposed in a function basis with degrees of freedom $\mathbf{v}$. Then with obvious notations the integration scheme for $\mathbf{s}^{i}$ on chord $i$ can be written as:

$$
\begin{aligned}
& \mathbf{s}^{i, 1}=\mathbf{s}_{0}^{i}, \\
& {\left[\mathbf{I}_{3}-\frac{h^{i, k}}{2} \boldsymbol{G}\left(\psi_{h}\left(\mathbf{x}^{i, k+1}\right), \mathbf{u}, \mathbf{v}\right)\right] \mathbf{s}^{i, k+1}} \\
& -\left[\mathbf{I}_{3}+\frac{h^{i, k}}{2} \boldsymbol{G}\left(\psi_{h}\left(\mathbf{x}^{i, k}\right), \mathbf{u}, \mathbf{v}\right)\right] \mathbf{s}^{i, k}=0, \quad k=1 \ldots N^{i}-1,
\end{aligned}
$$

which can be put into the more concise

$$
\boldsymbol{M}^{i}(\boldsymbol{\psi}, \mathbf{u}, \mathbf{v}) \mathbf{S}^{i}-\mathbf{S}_{0}^{i}=0
$$

where $\mathbf{M}^{i}$ is a $3 N^{i} \times 3 N^{i}$ band diagonal matrix, $\mathbf{S}^{i}=\left(\mathbf{s}^{i, 1}, \ldots, \mathbf{s}^{i, N^{i}}\right)$ is the vector of all Stokes vector states along chord $i$ and $\mathbf{S}_{0}^{i}=\left(\mathbf{s}_{0}^{i}, 0, \ldots 0\right)$ represents the initial conditions. 


\subsection{The discrete identification problem}

Using the discrete variables of the preceding section, cost function (18) can also be discretized leading to the following expression

$$
\begin{aligned}
& \left.J(\boldsymbol{\psi},\{\mathbf{S}\}, \mathbf{u}, \mathbf{v}):=\frac{1}{2} \| \boldsymbol{H} \boldsymbol{\psi}-\mathbf{m}\right) \|^{2} \\
& +\frac{1}{2}\left\|\boldsymbol{W}(\boldsymbol{\psi}) \mathbf{v}-\mathbf{N}_{e, o b s}\right\|^{2}+\sum_{i=1}^{N_{L}} \frac{1}{2}\left\|\boldsymbol{E} \mathbf{S}^{i}-\mathbf{s}_{o b s}^{i}\right\|^{2} \\
& +\frac{1}{2}\left\|\boldsymbol{R}_{u} \mathbf{u}\right\|^{2}+\frac{1}{2}\left\|\boldsymbol{R}_{v} \mathbf{v}\right\|^{2} .
\end{aligned}
$$

Here $\{\mathbf{S}\}$ is the vector $\left(\mathbf{S}^{1}, \ldots, \mathbf{S}^{N_{L}}\right)$. In order to lighten notations the $\frac{1}{\sigma}$ terms have been dropped and are assumed to be included in the observation operators and in the measurements. The linear observation operator $\boldsymbol{H}$ maps the finite element approximation $\boldsymbol{\psi}$ to the equivalent of magnetic measurements $\mathbf{m}$. The non-linear observation operator $\boldsymbol{W}(\boldsymbol{\psi}) \mathbf{v}$ represents the numerical quadrature of the electron density over the different chords. This term is linear in $\mathbf{v}$ but not in $\boldsymbol{\psi}$. The observation operator for Stokes vectors is given by matrix $\boldsymbol{E}$ such that $\boldsymbol{E S}^{i}=\mathbf{s}^{i, N^{i}}$ is the Stokes vector at the observation point. The last two terms involving matrices $\boldsymbol{R}_{u}$ and $\boldsymbol{R}_{v}$ are the discretization of the regularization terms in which we have gathered the contributions from functions $A$ and $B$.

The discrete identification problem can now be stated as

$$
\min _{\boldsymbol{\psi},\{\mathbf{S}\}, \mathbf{u}, \mathbf{v}} J(\boldsymbol{\psi},\{\mathbf{S}\}, \mathbf{u}, \mathbf{v})
$$

subject to the constraint of the model

$$
(\boldsymbol{A}+\boldsymbol{C}) \boldsymbol{\psi}-\mathbf{J}_{p}(\boldsymbol{\psi}, \mathbf{u})-\boldsymbol{L} \mathbf{I}=0
$$

and

$$
\boldsymbol{M}^{i}(\boldsymbol{\psi}, \mathbf{u}, \mathbf{v}) \mathbf{S}^{i}-\mathbf{S}_{0}^{i}=0, \quad i=1, \ldots N^{L} .
$$

Similarly to the continuous case we introduce Lagrange multipliers $\mathbf{p} \in \mathbb{R}^{N_{\psi}}$ and $\mathbf{Q}^{i} \in \mathbb{R}^{3 N^{i}}$ to formulate the Lagrangian for the optimization problem (34)- 
(36):

$$
\begin{aligned}
& \mathcal{L}(\boldsymbol{\psi},\{\mathbf{S}\}, \mathbf{u}, \mathbf{v}, \mathbf{p},\{\mathbf{Q}\})=J(\boldsymbol{\psi},\{\mathbf{S}\}, \mathbf{u}, \mathbf{v}) \\
& +\left(\mathbf{p} \cdot\left((\boldsymbol{A}+\boldsymbol{C}) \boldsymbol{\psi}-\mathbf{J}_{p}(\boldsymbol{\psi}, \mathbf{u})-\boldsymbol{L} \mathbf{I}\right)\right)+\sum_{i=1}^{N_{L}}\left(\mathbf{Q}^{i} \cdot\left(\mathbf{M}^{i}(\boldsymbol{\psi}, \mathbf{u}, \mathbf{v}) \mathbf{S}^{i}-\mathbf{S}_{0}^{i}\right)\right)
\end{aligned}
$$

and compute the discrete optimality system:

$$
\begin{gathered}
\boldsymbol{H}^{T}(\boldsymbol{H} \boldsymbol{\psi}-\mathbf{m})+D_{\psi}[\boldsymbol{W}(\boldsymbol{\psi}) \mathbf{v}]^{T}\left(\boldsymbol{W}(\boldsymbol{\psi}) \mathbf{v}-\mathbf{N}_{e, o b s}\right) \\
+\sum_{i=1}^{N_{L}} D_{\psi}\left[\boldsymbol{M}^{i}(\boldsymbol{\psi}, \mathbf{u}, \mathbf{v}) \mathbf{S}^{i}\right]^{T} \mathbf{Q}^{i}+\left(\boldsymbol{A}+\boldsymbol{C}-D_{\psi} \mathbf{J}_{p}(\boldsymbol{\psi}, \mathbf{u})\right)^{T} \mathbf{p}=0 \\
\boldsymbol{E}^{T}\left(\boldsymbol{E} \mathbf{S}^{i}-\mathbf{s}_{o b s}^{i}\right)+\boldsymbol{M}^{i}(\boldsymbol{\psi}, \mathbf{u}, \mathbf{v})^{T} \mathbf{Q}^{i}=0, \quad i=1, \ldots N_{L} \\
\boldsymbol{R}_{u}^{T} \boldsymbol{R}_{u} \mathbf{u}-D_{\mathbf{u}} \mathbf{J}_{p}(\boldsymbol{\psi}, \mathbf{u})^{T} \mathbf{p}+\sum_{i=1}^{N_{L}} D_{\mathbf{u}}\left[\boldsymbol{M}^{i}(\boldsymbol{\psi}, \mathbf{u}, \mathbf{v}) \mathbf{S}^{i}\right]^{T} \mathbf{Q}^{i}=0 \\
\boldsymbol{R}_{v}^{T} \boldsymbol{R}_{v} \mathbf{v}+\boldsymbol{W}(\boldsymbol{\psi})^{T}\left(\boldsymbol{W}(\boldsymbol{\psi}) \mathbf{v}-\mathbf{N}_{e, o b s}\right)+\sum_{i=1}^{N_{L}} D_{\mathbf{v}}\left[\boldsymbol{M}^{i}(\boldsymbol{\psi}, \mathbf{u}, \mathbf{v}) \mathbf{S}^{i}\right]^{T} \mathbf{Q}^{i}=0 \\
(\boldsymbol{A}+\boldsymbol{C}) \boldsymbol{\psi}-\mathbf{J}_{p}(\boldsymbol{\psi}, \mathbf{u})-\boldsymbol{L} \mathbf{I}=0 \\
\boldsymbol{M}^{i}(\boldsymbol{\psi}, \mathbf{u}, \mathbf{v}) \mathbf{S}^{i}-\mathbf{S}_{0}^{i}=0, \quad i=1, \ldots N^{L}
\end{gathered}
$$

Equation (38) is the discrete adjoint equilibrium problem for p. Equation (39) is the adjoint Stokes equation on each chord for $\mathbf{Q}^{i}$. The transposed operator corresponds to the backwards integration from $Z_{1}^{i}$ to $Z_{0}^{i}$ of the adjoint Stokes equation. Equations 40 and 41 give the gradient of the cost function seen as a function of $(\mathbf{u}, \mathbf{v})$ only, when the model constraint is satisfied. The last two equations are the model constraint.

The optimality system $38-43$ is a system of non-linear equations $\mathbf{F}(\mathbf{X})=0$ with $\mathbf{X}=(\boldsymbol{\psi},\{\mathbf{S}\}, \mathbf{u}, \mathbf{v}, \mathbf{p},\{\mathbf{Q}\})$. We solve it thanks to a Newton-type method. If the second order derivative terms are neglected in the computation of the Jacobian of the optimality system, $\boldsymbol{K}(\mathbf{X}) \approx D_{\mathbf{X}} \mathbf{F}(\mathbf{X})$, the following approximated Newton iteration is obtained

$$
\boldsymbol{K}\left(\mathbf{X}^{k}\right)\left(\mathbf{X}^{k+1}-\mathbf{X}^{k}\right)=-\mathbf{F}\left(\mathbf{X}^{k}\right)
$$


where

$$
\begin{aligned}
& K=
\end{aligned}
$$

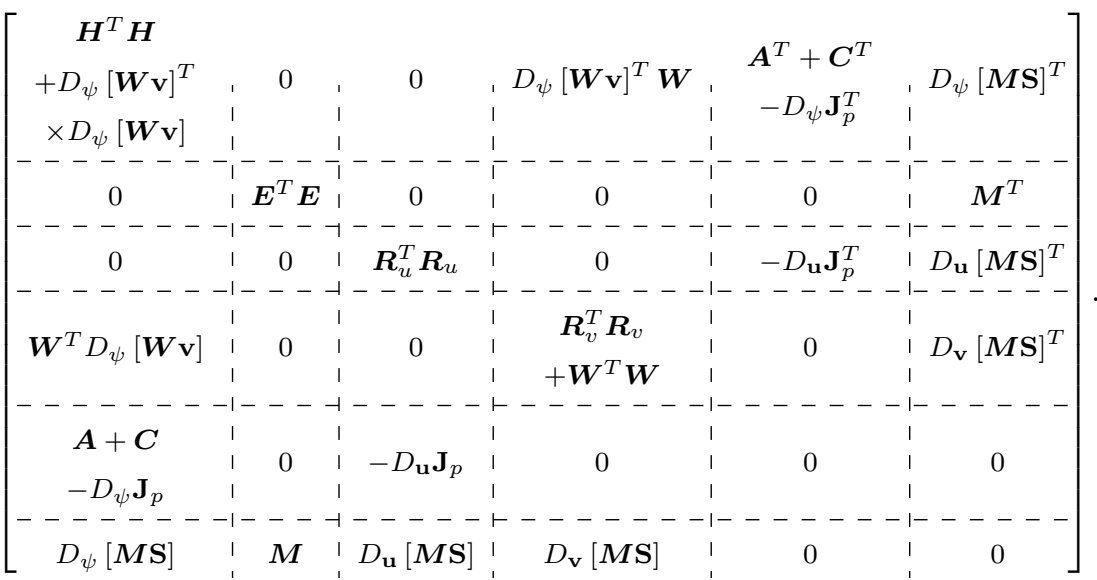

Here all the dependances in $\boldsymbol{\psi}, \mathbf{u}$ or $\mathbf{v}$ have been dropped to lighten notations and we have defined

$$
M=\left[\begin{array}{cccc}
M^{1} & 0 & \ldots & 0 \\
& \ddots & & \\
& & \ddots & \\
0 & \ldots & 0 & M^{N_{L}}
\end{array}\right]
$$

as well as

$$
D_{\psi}[\mathbf{M S}]=\left[\begin{array}{c}
D_{\psi}\left[\boldsymbol{M}^{1} \mathbf{S}^{1}\right] \\
\vdots \\
D_{\psi}\left[\boldsymbol{M}^{N_{L}} \mathbf{S}^{1}\right]
\end{array}\right]
$$

and $D_{\mathbf{u}}[\boldsymbol{M S}]$ and $D_{\mathbf{v}}[\boldsymbol{M S}]$ are similarly defined.

The approximated Newton iterative scheme (44) presented here is the adaptation to the particular case of tokamak equilibrium reconstruction using magnetic and Stokes vector measurements of PDE-constrained optimization methods [40] and falls into the family of sequential quadratic programming (SQP) methods 30]. The algorithm presented here can be modified to consider the cases where only magnetic measurements or polarimetry measurements using classical formulation (17) are used. 
One approximated Newton iteration for the optimality system corresponds exactly to the minimization of a quadratic functional ( $J$ in Eq. 33 in which the $\boldsymbol{W}(\boldsymbol{\psi}) \mathbf{v}$ term is linearized at $\left.\left(\boldsymbol{\psi}^{k}, \mathbf{v}^{k}\right)\right)$ under linear constraints (model equations 35 and 36 linearized at $\left.\left(\boldsymbol{\psi}^{k},\{\mathbf{S}\}^{k}, \mathbf{u}^{k}, \mathbf{v}^{k}\right)\right)$. This is the point of view adopted in [6, 20] where at each iteration (called external iteration) the linear quadratic control problem can be solved by a sequence of internal conjugate gradient iterations. This approach is useful if system (44) becomes too large for the linear solver (e.g. in case of very small discretization steps). However with modern computers, performant linear solver libraries and reasonable discretization parameters, this resolution is possible and fast. The numerical results presented in the next section are obtained with the iterative scheme (44) directly solving the optimality system.

Similarly to Newton method used for the resolution of the direct equilibrium problem of Eq. (30), the performance of the SQP method used for the resolution of the identification problem relies on the accuracy of the derivative terms $D_{\psi} \mathbf{J}_{p}$, $D_{\mathbf{u}} \mathbf{J}_{p}, D_{\psi}[\boldsymbol{M S}], D_{\mathbf{u}}[\boldsymbol{M S}]$ and $D_{\mathbf{v}}[\boldsymbol{M S}]$. In this work we have implemented the exact derivatives of the fully discretized operators. This essential but very technical work is not further detailed here.

\section{Numerical experiments}

The numerical methods presented in the previous section have been implemented in the code FEEQS.M which is a Matlab implementation of the methods for free-boundary equilibrium computations presented in [35]. The ITER geometry and magnetic sensors (145 B probes and 4 flux loops) positions are taken from the European Integrated Tokamak Modeling database 41, 42. The 15 polarimetry viewing chords are taken from [43].

The general methodology is the following. Synthetic measurements are generated integrating the direct equilibrium and Stokes models, given reference functions $A, B$ and $N_{e}$. Figures 2 and 3 show an example of such an equilibrium as well as the magnetic sensors and the polarimetry chords. In all simulations 
the scaling factor $\lambda$ in the current density (7) is computed such that the total plasma current is $I_{p}=15[M A]$. The vacuum toroidal field is $B_{0}=5.3[T]$ and $r_{0}=6.2[\mathrm{~m}]$.

A wavelength of $l=0.195[\mathrm{~mm}]$ is assumed for the laser beams and the associated values for constants appearing in Stokes equation are $C_{1}=2.42 \times$ $10^{-20} l^{3}=1.7944 \times 10^{-22}\left[\mathrm{~m}^{2} \mathrm{~T}^{-2}\right]$ and $C_{3}=5.23 \times 10^{-19} l^{2}=1.9887 \times 10^{-20}$ $\left[m^{2} T^{-1}\right]$. Following [24 the initial Stokes vectors are chosen as $\mathbf{s}_{0}^{i}=(0,1,0)$.

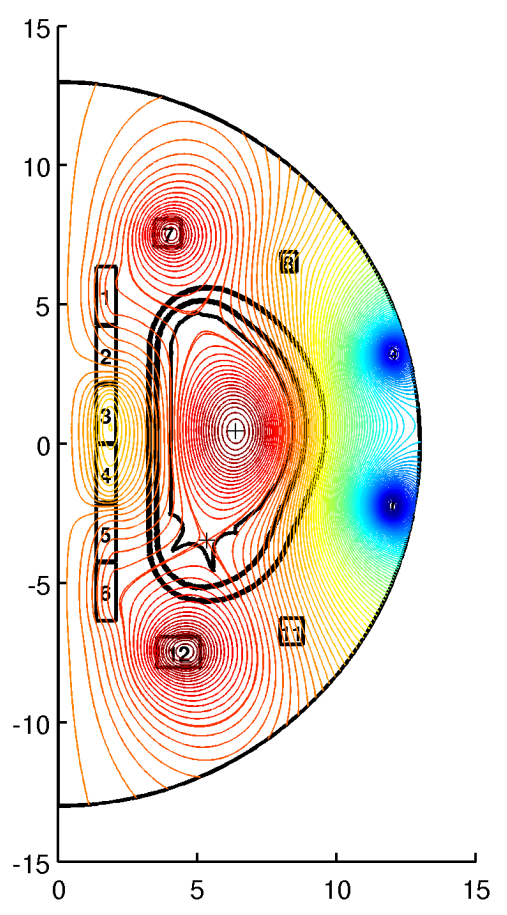

Figure 2: Computed ITER equilibrium for TC1. Isoflux contours are shown on the full computation domain. The poloidal field coils appear numbered from 1 to 12 . The vacuum vessel and limiter contours are also shown.

Then in a second step these measurements are plugged in cost function (33) and the optimization problem is solved using the iterative algorithm (44) presented above. The initial guess for this resolution consists in a given circular plasma boundary in which the flux $\psi$ is a constant and outside of which it is 0 , as well as affine functions $A(x)=B(x)=1-x$. A first Newton iteration for the 


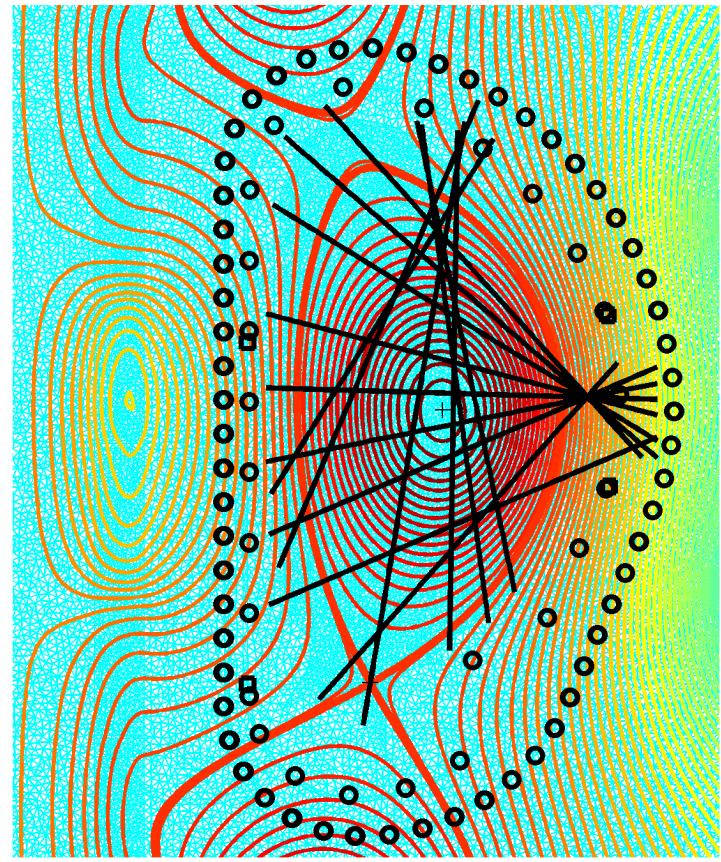

Figure 3: Zoom on the vacuum vessel for computed ITER equilibrium TC1. Isoflux contours are shown, the thick one corresponds to the value $\psi_{b}$ of the plasma boundary defined by the presence of an X-point. The finite element mesh is visible in the background. The small black circles indicate the position of the $145 \mathrm{~B}$ probes. The 4 small squares indicate the position of the 4 flux loops. The black segments represent the 15 chords used for intereferometry and polarimetry computations.

direct equilibrium problem with fixed $I_{p}[35]$ is performed in order to compute a first $\psi$ map and to give a value to the scaling factor $\lambda$. Thereon this factor is kept fixed and for what concerns the plasma current density only $\mathbf{u}$ representing the functions to be identified evolves during the iterations. Convergence is assumed when

$$
\left\|\mathbf{X}^{k+1}-\mathbf{X}^{k}\right\| /\left\|\mathbf{X}^{k}\right\|<10^{-12}
$$

which takes about 10 iterations to be satisfied.

Three types of experiments are conducted: type $\mathrm{M}$ in which only magnetics are used; type MF in which magnetics, interferometry and classical Faraday measurements are used; and type MS in which magnetics, interferometry and 
Stokes vector measurements are used.

The finite element mesh is composed of $N_{\psi}=30449$ nodes among which 30270 correspond to free values of $\psi$ (the remaining correspond to the imposed boundary condition $\psi=0$ on the axis $r=0$ ). The discretization step on each chord is chosen to be $h=0.05[\mathrm{~m}]$ giving a vector $\{\mathbf{S}\}$ of size $3 \times 2022$. Each function to be identified is decomposed in 11 cubic splines defined on $[0,1]$ with knots at $0,0.1, \ldots, 1$. Therefore $X$ is a vector of size $2 \times 30270+2 \times 11=$ 60562 for type $\mathrm{M}$ experiments, $2 \times 30270+3 \times 11=60573$ for type MF and $2 \times(30270+3 \times 2022)+3 \times 11=72705$ for type MS experiments.

We consider 2 test cases (TC). In TC1, the reference functions are given analytically as $A(x)=B(x)=\left(1-x^{1.5}\right)^{0.9}$ and $N_{e}(x)=N_{0}\left(1-x^{3}\right)$ with $N_{0}=0.5\left[10^{20} m^{-3}\right]$. In TC2 the $A$ and $B$ profiles are provided point by point and do not have an analytic expression. The electron density is $N_{e}(x)=N_{1}+\left(N_{0}-N_{1}\right)\left(1-x^{3}\right)$ with $N_{1}=0.01\left[10^{20} m^{-3}\right]$. For both cases the caracteristic profiles $A, B$ and $N_{e}$ can be seen on Fig. 4 and 5 respectively. The flux surface averaged [6] current density $\left\langle j_{\phi} / r\right\rangle$, safety factor $q$ and pressure $p$ profiles are shown on Fig. 6 and 7 respectively. One should notice that if on the one hand the $A$ and $B$ profiles in TC1 are quite academic on the other hand in TC2 they are much more challenging in term of variations and feature non zero values at the plasma boundary $\psi_{\mathrm{N}}=1$. For TC2 the plasma is in an H-mode configuration.

For each numerical experiment the regularization parameters $\varepsilon$ and $\varepsilon_{N_{e}}$ are tuned to their lowest value, typically $10^{-3}-10^{-2}$, avoiding oscillations in the reconstructed profiles or non convergence of the code.

As a first step and in order to validate the good convergence properties of the code we first consider the case of perfect measurements (without additional noise). In all experiments the relative errors between measurements and reconstructed values is of order $10^{-5}$ for magnetics and $10^{-4}$ for interferometry and polarimetry indicating an excellent fit. The relative error on $\psi$ is also very small and the plasma boundary is perfectly recovered. The $N_{e}$ profile is very well recovered. Profiles $A$ and $B$ are also well recovered in the 3 experiments 
M, MF and MS even though the discrepancies between the real reference profile values and the reconstructed ones near $\psi_{\mathrm{N}}=0$ (the magnetic axis) tend to be larger than close to $\psi_{\mathrm{N}}=1$ (the plasma boundary). This is typical for this plasma current identification problem [15]. The main point here is that the use of Faraday measurements or of Stokes vector measurements decreases this error. In this perfect data experiment the difference between MF and MS is however barely distinguishable. It is clearer when noise is added.

We add a $1 \%$ noise on magnetics and a $10 \%$ noise on interferometry and polarimetry measurements and conduct a new set of identification experiments.

Table 1 shows the mean relative errors between real values of measured quantities (i.e measurements without noise) and reconstructed values. They are below 1\% for magnetics and 10\% for interferometry and polarimetry which shows the benefit of the regulariation term on the ill-posedness of the problem and the quality of the reconstruction. The resulting errors on $\psi$ are very small and the plasma boundary is perfectly recovered for both TC1 and TC2.

\begin{tabular}{c|c|c|c|c|c|c|c|} 
& & B probes & flux loops & interfero. & \multicolumn{3}{|c|}{ polarimetry } \\
\hline \multirow{2}{*}{ M } & TC1 & 0.0569 & 0.2505 & & & & \\
& TC2 & 0.1055 & 0.0939 & & & & \\
\hline \multirow{2}{*}{ MF } & TC1 & 0.0515 & 0.2511 & 1.9224 & 1.9966 & & \\
& TC2 & 0.1083 & 0.1156 & 1.2835 & 2.5153 & & \\
\hline \multirow{2}{*}{ MS } & TC1 & 0.0454 & 0.2074 & 0.2746 & 1.4575 & 1.1761 & 1.5625 \\
& TC2 & 0.0845 & 0.1368 & 0.2355 & 1.4540 & 2.4148 & 2.0729 \\
\hline
\end{tabular}

Table 1: Mean relative errors $\left(\times 10^{2}\right)$ between real values of measured quantities (i.e measurements without noise) and reconstructed values for TC1 and TC2, with noisy measurements ( $1 \%$ on magnetics and $10 \%$ on interferometry and polarimetry).

The sensor by sensor measurements, error bars and values computed by the reconstruction code are also shown on Figs. 8 and 9 for TC1 and TC2 respectively. The fit to measurements is of excellent quality. In these last figures, the comparison between the Faraday measurements plot and the first Stokes vector component, $s_{1}$, measurements plot as well the investigation of the 
second Stokes vector component, $s_{2}$, measurements plot show that the plasma is in a state where the Stokes model cannot be approximated with the usual Faraday measurement equation. If this approximation were valid then we should observe with the chosen initial conditions for the Stokes vector, $\mathbf{s}_{0}=(0,1,0)$, that the Faraday measurement verifies $\alpha \approx-\frac{1}{2} s_{1}$ and that $s_{2} \approx 1$ [27, page R71].

The reconstructed $A, B$ and $N_{e}$ profiles for the different experiments (M, $\mathrm{MF}$ and MS) are shown on Fig. 4 and 5 for TC1 and TC2 respectively. The error profiles, normalized by the largest error value, are also shown in the right column of these figures. The same is plotted for the $\left\langle j_{\phi} / r>, q\right.$ and $p$ profiles on Fig. 6 and 7. It is clearly observed that the MS reconstructed profiles are better than the MF ones which are also better than the M ones. This is confirmed by Fig. 14 which shows the computed relative error in $L^{2}$ norm between the reconstructed and reference profiles.

This was expected since more measurements are used to constrain the equilibrium. The use of Stokes vector measurements improves the identification of profiles $A$ and $B$ compared to the use of Faraday measurements and of magnetic measurements alone, particularly in the magnetic axis region. The other reconstructed profiles benefit from this improvement. For example the safety factor value at the axis is better reconstructed.

Finally this improvement can also be observed on 2D plots of $\psi$ (Fig. 10 for TC1 and Fig. 11 for TC2) and $\left\|\mathbf{B}_{p}\right\|$ (Fig. 12 for TC1 and Fig. 13 for TC2) in $\Omega_{\mathrm{L}}$ the domain accessible to the plasma. The errors between reference and reconstructed values are clearly decreasing while going from $\mathrm{M}$ to MF and to MS.

\section{Conclusion}

In this work a numerical method enabling the consistent resolution of the inverse equilibrium reconstruction problem in the framework of non-linear freeboundary equilibrium coupled to the Stokes model equation for polarimetry is 
provided. Using optimal control theory, the optimality system for this inverse problem in which the direct model consists in a non-linear partial differential equations and a set of systems of ordinary differential equation is derived. An $\mathrm{SQP}$ method is proposed for the numerical resolution of the problem. Numerical experiments show that the method is efficient. However the complexity of the model considered here has encouraged to formulate explicitely the adjoint model for the resolution of the inverse problem. This is expensive from the computational point of view and might not be suited for real-time reconstruction.

In addition, the numerical solutions for two test cases in an ITER configuration show that the profiles recovery clearly benefits from the use of Stokes vector measurements compared to the classical case of Faraday measurements. This is not surprising as the Stokes vector constraints add more information to the inverse problem. The benefit should be even more interesting with real experimental measurements which are expected, according to the literature, to be more accurately represented by the Stokes model in ITER-like plasma regimes.

\section{Acknowledgment}

We would like to thank Mireille Schneider (ITER) and Jean-Francois Artaud (CEA) for their help in providing the $p^{\prime}$ and $f f^{\prime}$ profiles for Test Case TC2.

We would also like to thank the two anonymous reviewers for their numerous remarks and constructive criticism from which the paper has benefitted significantly. 

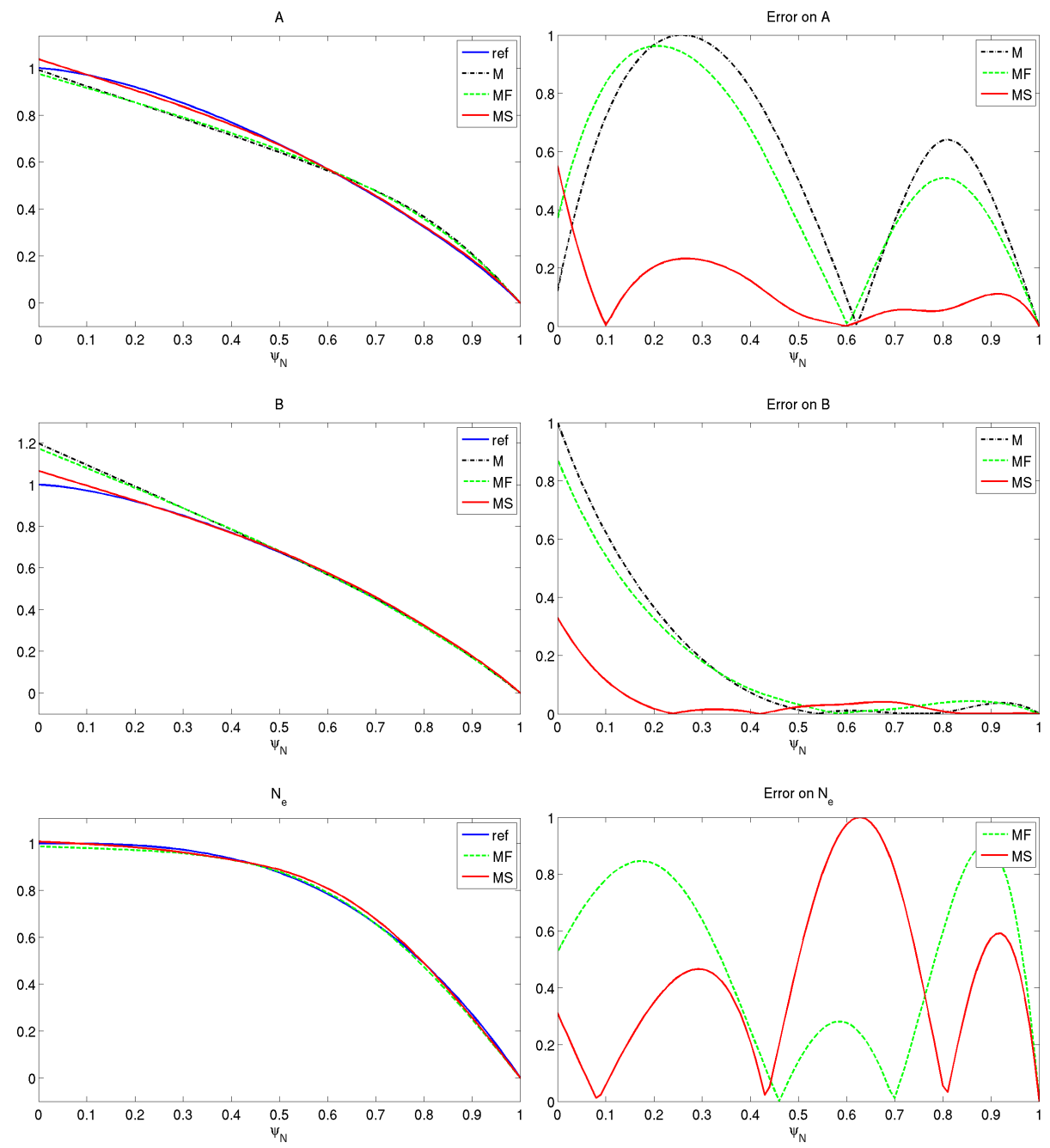

Figure 4: Profiles $A, B$ and $N_{e}$ for TC1. Left column: reference $A, B$ and $N_{e}$ profiles and the reconstructed ones for experiments M, MF and MS with $1 \%$ noise on magnetics and $10 \%$ noise on interferometry and polarimetry. Right column: error, between reconstructed and reference profiles, normalized by the largest error value. 

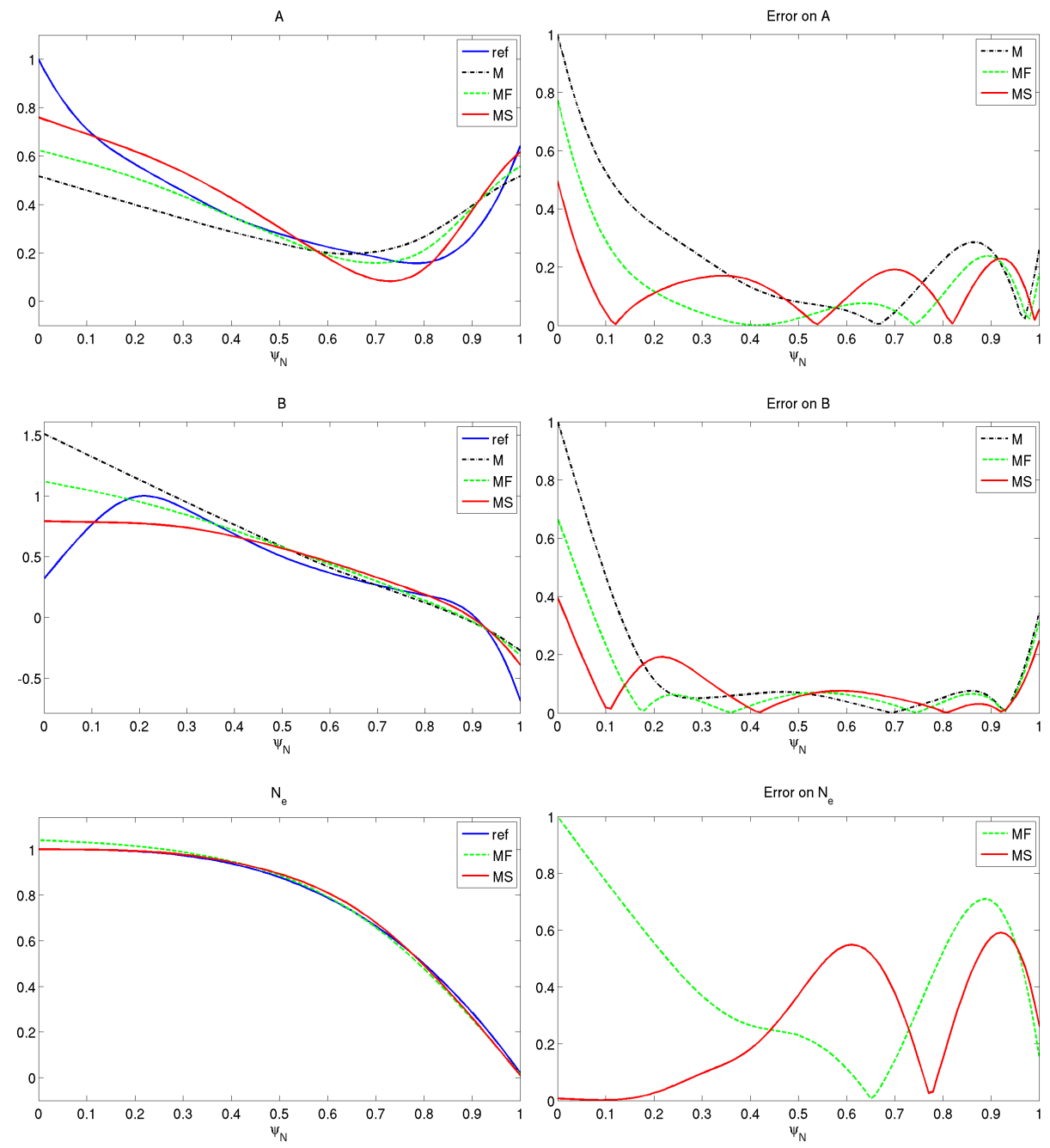

Figure 5: Profiles $A, B$ and $N_{e}$ for TC2. Left column: reference $A, B$ and $N_{e}$ profiles and the reconstructed ones for experiments M, MF and MS with $1 \%$ noise on magnetics and $10 \%$ noise on interferometry and polarimetry. Right column: error, between reconstructed and reference profiles, normalized by the largest error value. 

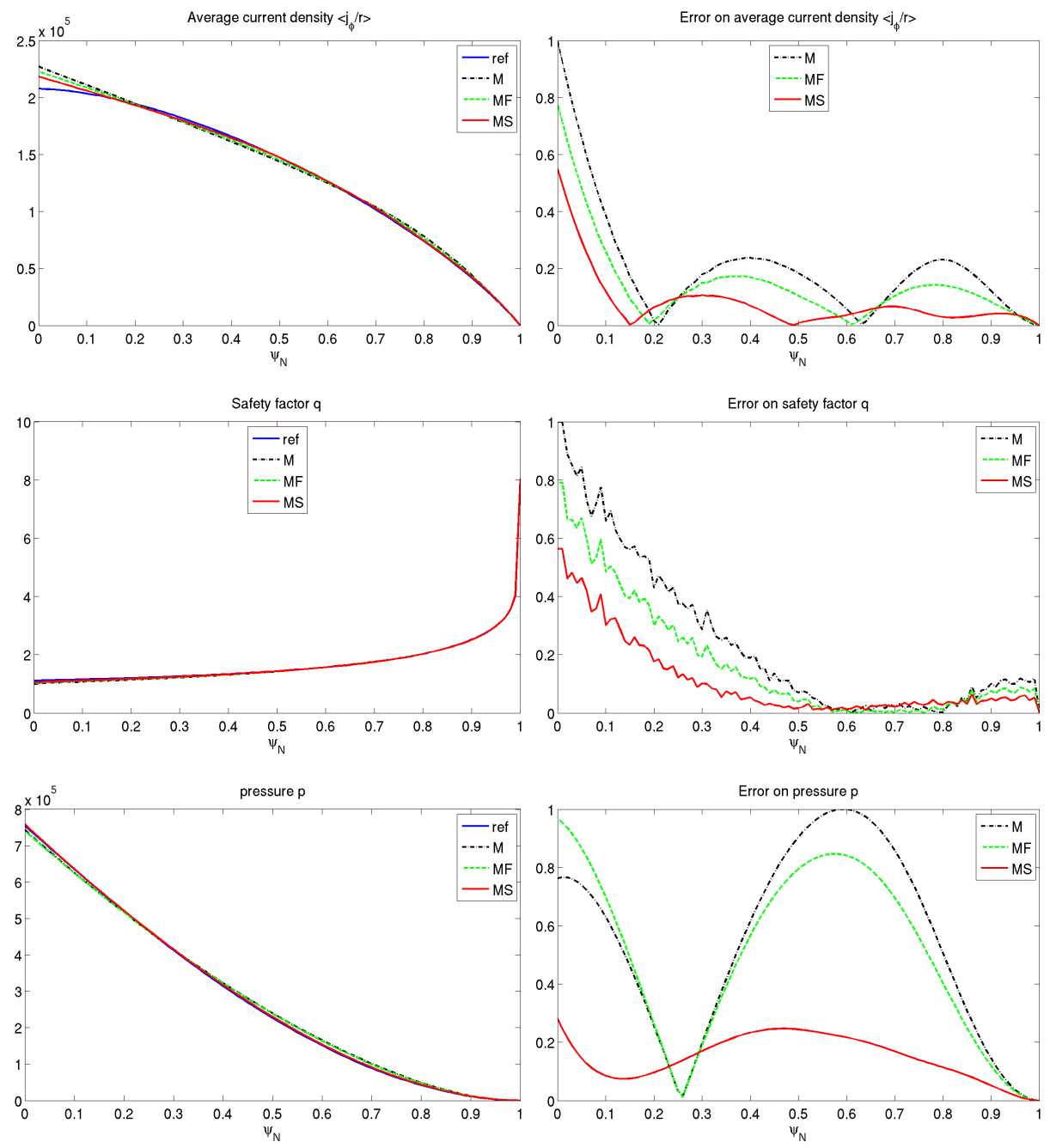

Figure 6: Profiles $\left\langle j_{\phi} / r\right\rangle, q$ and $p$ for TC1. Left column: reference $\left\langle j_{\phi} / r\right\rangle, q$ and $p$ profiles and the reconstructed ones for experiments M, MF and MS with $1 \%$ noise on magnetics and $10 \%$ noise on interferometry and polarimetry. Right column: error, between reconstructed and reference profiles, normalized by the largest error value. 

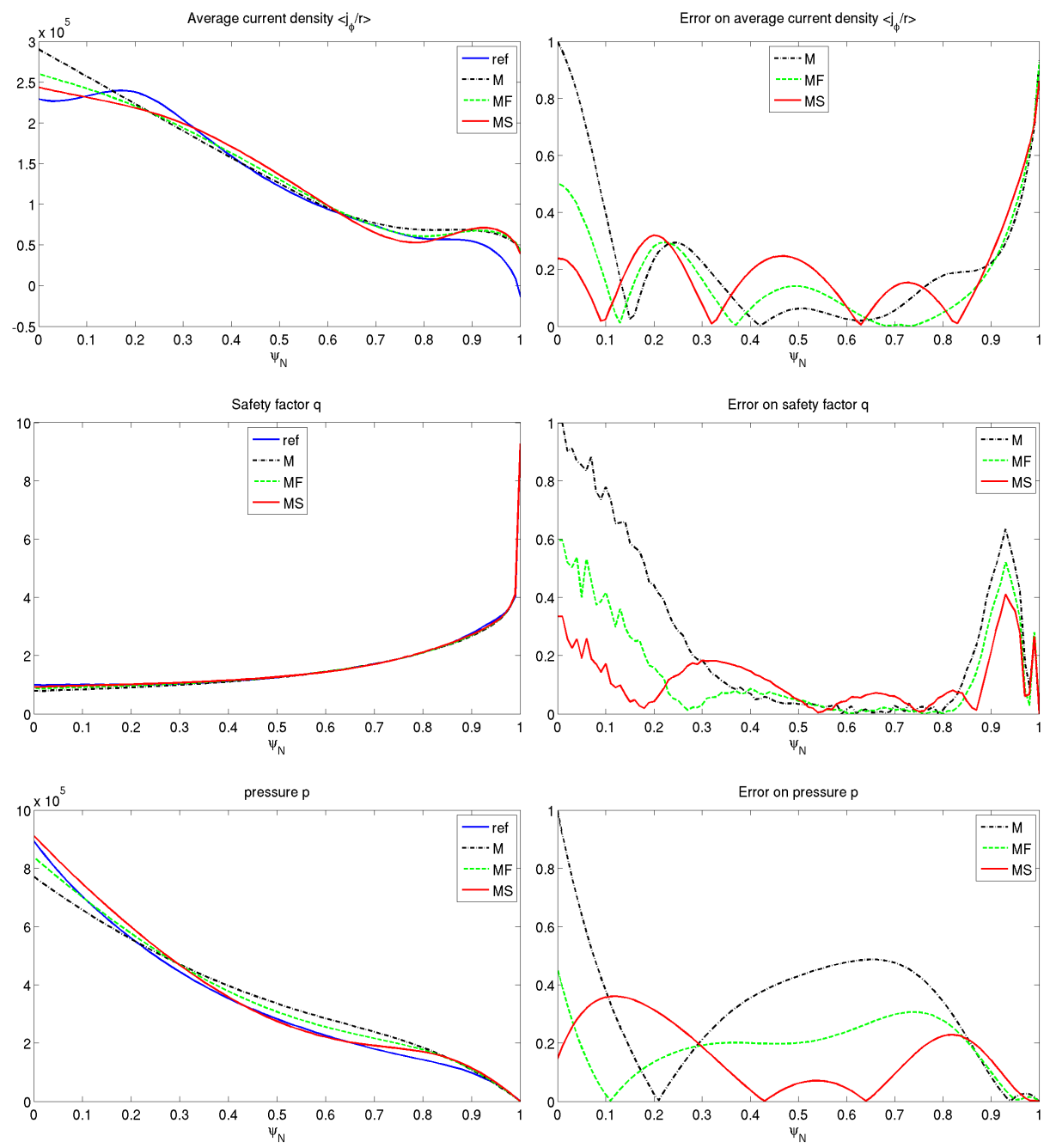

Figure 7: Profiles $\left\langle j_{\phi} / r\right\rangle, q$ and $p$ for TC2. Left column: reference $\left\langle j_{\phi} / r\right\rangle, q$ and $p$ profiles and the reconstructed ones for experiments M, MF and MS with $1 \%$ noise on magnetics and $10 \%$ noise on interferometry and polarimetry. Right column: error, between reconstructed and reference profiles, normalized by the largest error value. 

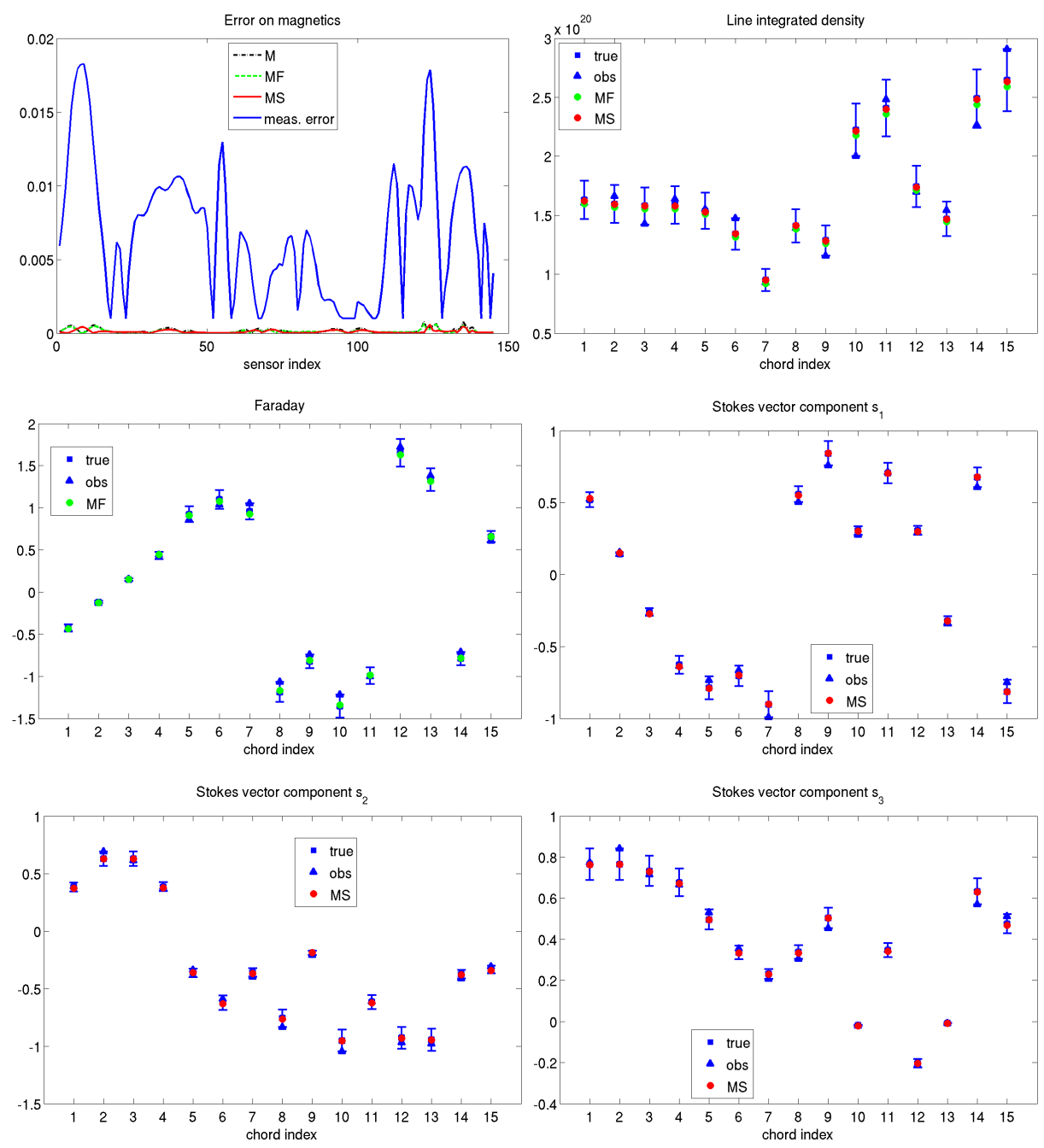

Figure 8: Measurements plots for TC1 with $1 \%$ noise on magnetics and $10 \%$ noise on interferometry and polarimetry. Top left: absolute errors in $[T]$ on reconstucted magnetics, far below the measurement error in blue. Top right: line integrated density measurements, true values and their error bars, observed noisy values and reconstructed values. Middle left: same for Faraday rotation measurements. Middle right and bottom row: same for Stokes vector components measurements. 

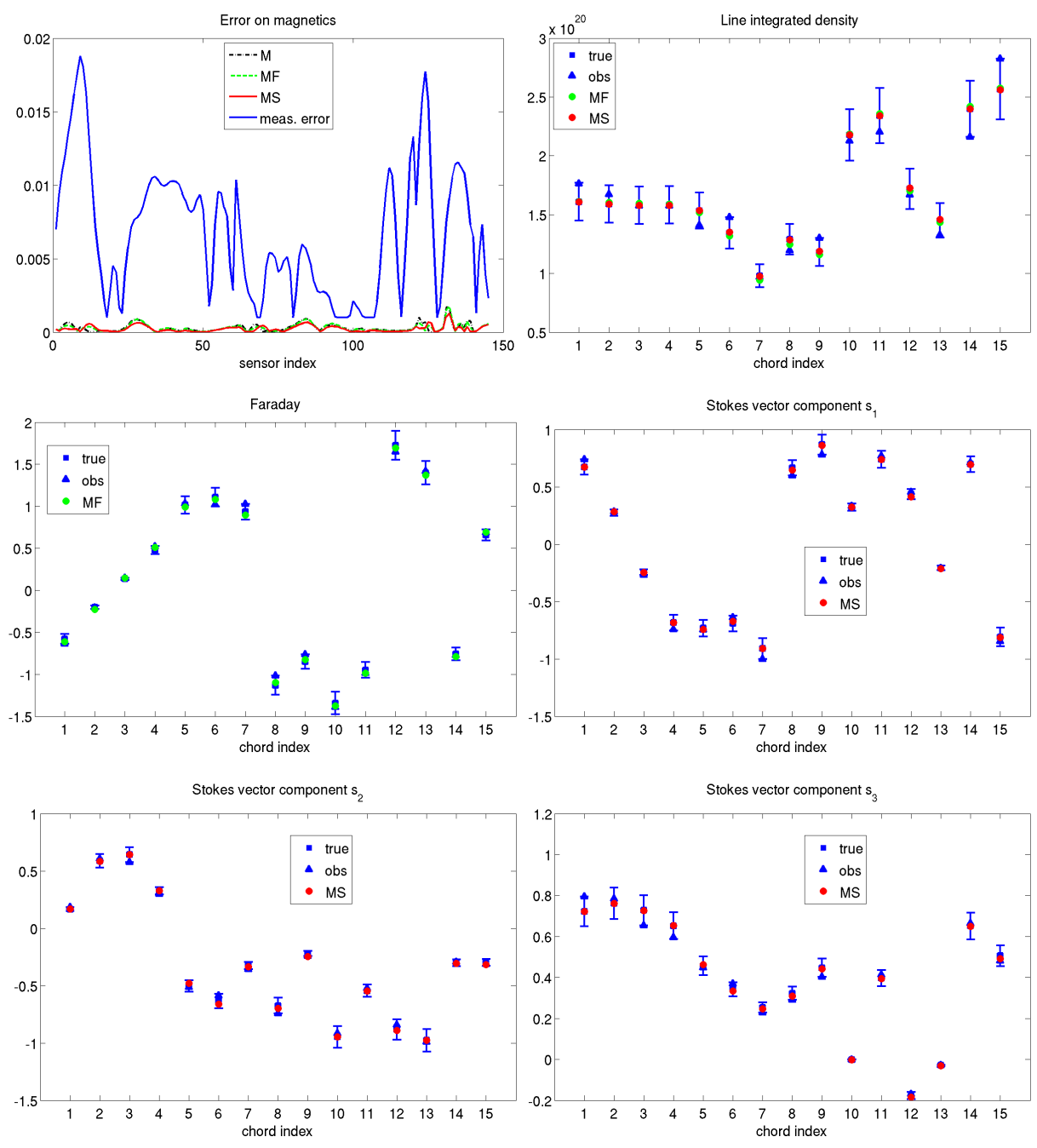

Figure 9: Measurements plots for TC2 with $1 \%$ noise on magnetics and $10 \%$ noise on interferometry and polarimetry. Top left: absolute errors in $[T]$ on reconstucted magnetics, far below the measurement error in blue. Top right: line integrated density measurements, true values and their error bars, observed noisy values and reconstructed values. Middle left: same for Faraday rotation measurements. Middle right and bottom row: same for Stokes vector components measurements. 


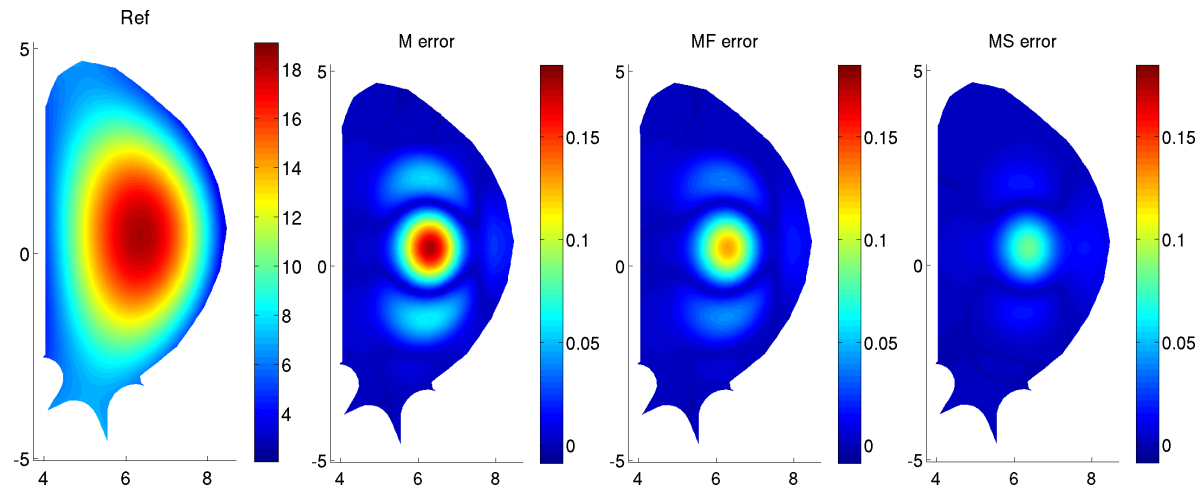

Figure 10: 2D $\psi$ maps for TC1. Left: reference $\psi$ map in $\Omega_{\mathrm{L}}$. Then from left to right: absolute error between reconstructed and reference $\psi$ map for experiments M, MF and MS with $1 \%$ noise on magnetics and $10 \%$ noise on interferometry and polarimetry.

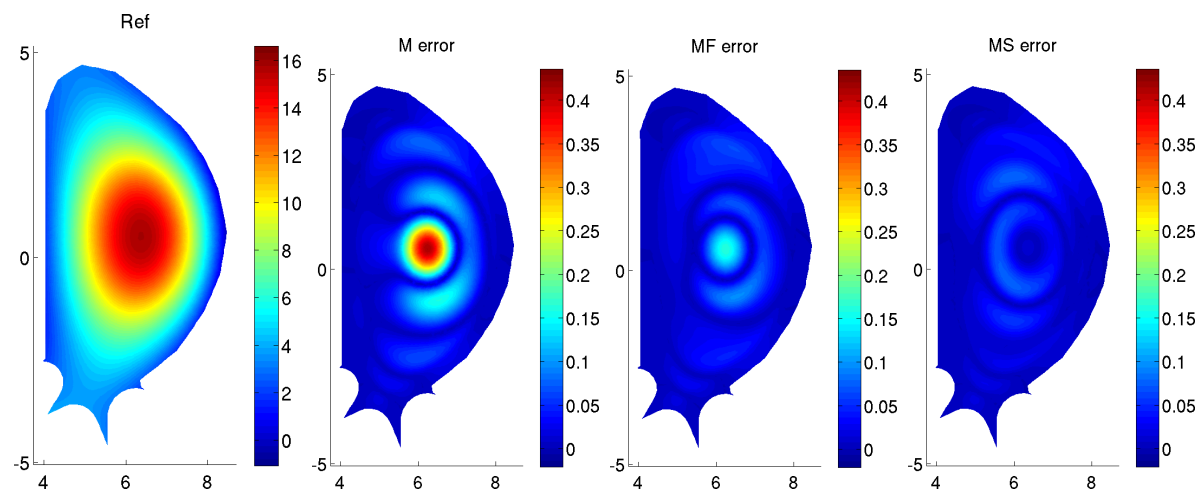

Figure 11: 2D $\psi$ maps for TC2. Left: reference $\psi$ map in $\Omega_{\mathrm{L}}$. Then from left to right: absolute error between reconstructed and reference $\psi$ map for experiments M, MF and MS with $1 \%$ noise on magnetics and $10 \%$ noise on interferometry and polarimetry. 


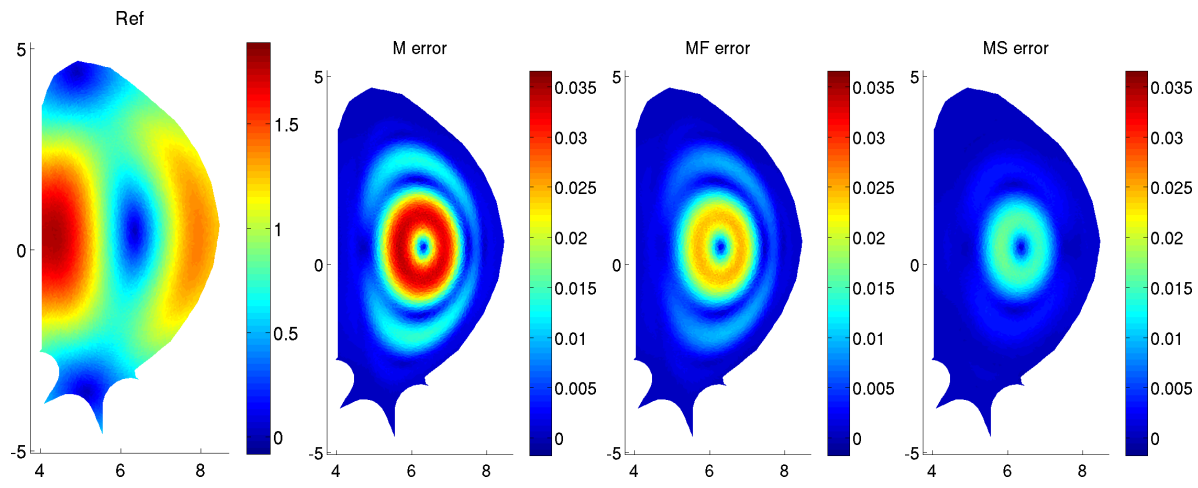

Figure 12: 2D $\left\|\mathbf{B}_{p}\right\|$ maps for TC1. Left: reference $\left\|\mathbf{B}_{p}^{\text {ref }}\right\|$ map in $\Omega_{\mathrm{L}}$. Then from left to right: absolute error between reconstructed and reference, $\left\|\mathbf{B}_{p}-\mathbf{B}_{p}^{\text {ref }}\right\|$ map for experiments $\mathrm{M}, \mathrm{MF}$ and MS with $1 \%$ noise on magnetics and $10 \%$ noise on interferometry and polarimetry.

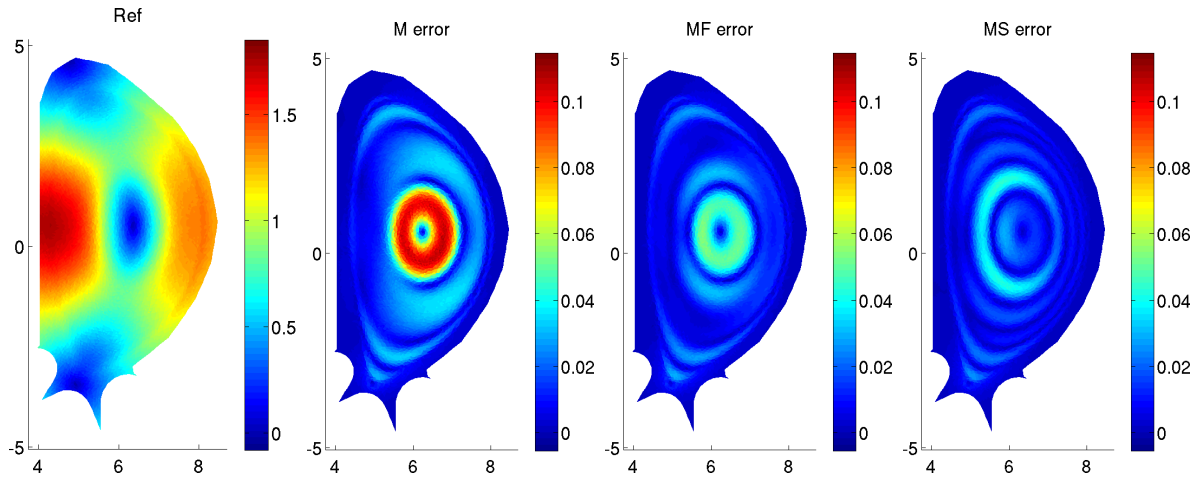

Figure 13: 2D $\left\|\mathbf{B}_{p}\right\|$ maps for TC2. Left: reference $\left\|\mathbf{B}_{p}^{r e f}\right\|$ map in $\Omega_{\mathrm{L}}$. Then from left to right: absolute error between reconstructed and reference, $\left\|\mathbf{B}_{p}-\mathbf{B}_{p}^{r e f}\right\|$ map for experiments $\mathrm{M}, \mathrm{MF}$ and MS with $1 \%$ noise on magnetics and $10 \%$ noise on interferometry and polarimetry. 


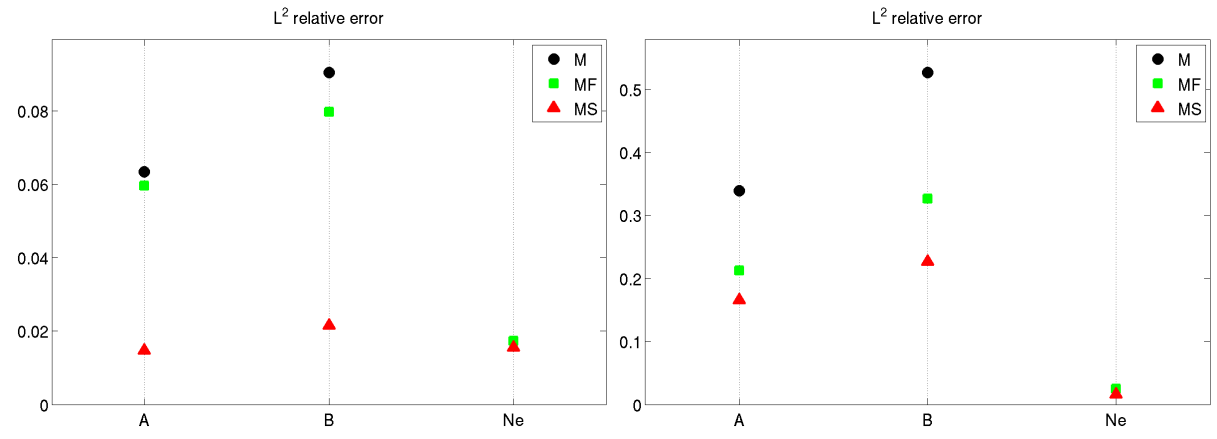

Figure 14: Relative error between reconstructed and reference profile for $A, B$ and $N_{e}$ in $L^{2}$ norm for experiments $\mathrm{M}, \mathrm{MF}$ and $\mathrm{MS}$, with $1 \%$ noise on magnetics and $10 \%$ noise on interferometry and polarimetry. Left: TC1. Right: TC2. 


\section{References}

[1] V. D. Shafranov, Plasma Physics 13 (1971) 757.

[2] L. Zakharov, V. Shafranov, Sov. Phys. Tech. Phys. 18 (1973) 151-156.

[3] J. Luxon, B. Brown, Nuclear Fusion 22 (1982) 813-821.

[4] D. Swain, G. Neilson, Nuclear Fusion 22 (1982) 1015-1030.

[5] L. Lao, Nuclear Fusion 25 (1985) 1421.

[6] J. Blum, Numerical Simulation and Optimal Control in Plasma Physics with Applications to Tokamaks, Series in Modern Applied Mathematics, Wiley Gauthier-Villars, Paris, 1989.

[7] H. Grad, H. Rubin, Proceedings of the 2nd UN Conf. on the Peaceful Uses of Atomic Energy 31 (1958) 190. URL: http://www-naweb.iaea.org/napc/physics/2ndgenconf/data/ Proceedings1958/papersVol31/Paper25_Vol31.pdf.

[8] V. Shafranov, Soviet Journal of Experimental and Theoretical Physics 6 (1958) 545. URL: http://adsabs.harvard.edu/abs/1958JETP ...6. $.545 \mathrm{~S}$

[9] R. Lüst, A. Schlüter, Z. Naturforsch. A 12 (1957) 850-854.

[10] L. Lao, J. Ferron, R. Geoebner, W. Howl, H. St. John, E. Strait, T. Taylor, Nuclear Fusion 30 (1990) 1035.

[11] J. Blum, E. Lazzaro, J. O’Rourke, B. Keegan, Y. Stefan, Nuclear Fusion 30 (1990) 1475.

[12] P. J. Mc Carthy, Physics of Plasmas 6 (1999) 3554-3560. URL: http://scitation.aip.org/content/aip/journal/pop/6/9/10.1063/ 1.873630, doi:http://dx.doi.org/10.1063/1.873630.

[13] W. Zwingmann, Nuclear Fusion 43 (2003) 842-850. 
[14] J. Blum, C. Boulbe, B. Faugeras, in: Journal of Physics: Conference Series. Proceedings of the 6th International Conference on Inverse Problems in Engineering: Theory and Practice, volume 135, IOP Publishing, 2008, p. 012019 .

[15] J. Blum, C. Boulbe, B. Faugeras, Journal of Computational Physics 231 (2012) 960 - 980. URL: http://www.sciencedirect.com/science/ article/pii/S0021999111002294. doi/http://dx.doi.org/10.1016/j. jcp.2011.04.005.

[16] B. Faugeras, J. Blum, C. Boulbe, P. Moreau, E. Nardon, Plasma Phys. Control Fusion 56 (2014) 114010.

[17] J.-M. Moret, B. Duval, H. Le, S. Coda, F. Felici, H. Reimerdes, Fusion Eng. Design 91 (2015) 1-15. URL: http://www.sciencedirect.com/ science/article/pii/S0920379614005973. doi/http://dx.doi.org/ $10.1016 / \mathrm{j}$. fusengdes.2014.09.019

[18] V. Pustovitov, Nuclear Fusion 41 (2001) 721.

[19] F. Hofmann, G. Tonetti, Nuclear Fusion 28 (1988) 1871. URL: http://iopscience.iop.org/0029-5515/28/10/014/pdf/0029-5515_ 28_10_014.pdf.

[20] J. Blum, H. Buvat, in: Biegler, Coleman, Conn, Santosa (Eds.), IMA Volumes in Mathematics and its Applications, Volume 92, "Large Scale Optimization with applications", Part 1: Optimization in inverse problems an design, Springer, New York, 1997, pp. 17-36.

[21] V. Mirnov, W. Ding, D. Brower, M. Van Zeeland, T. Carlstrom, Phys. Plasmas 14 (2007) 102105.

[22] F. Orsitto, A. Boboc, C. Mazzotta, E. Giovannozzi, L. Zabeo, JET EFDA Contributors, Plasma Phys. Control Fusion 50 (2008) 115009. 
[23] F. Orsitto, A. Boboc, P. Gaudio, M. Gelfusa, E. Giovannozzi, C. Mazzotta, A. Murari, JET EFDA Contributors, Rev. Sci. Instrum. 81 (2010) 10D533.

[24] F. Orsitto, A. Boboc, P. Gaudio, M. Gelfusa, E. Giovannozzi, C. Mazzotta, A. Murari, JET EFDA Contributors, Plasma Phys. Control Fusion 53 (2011) 035001.

[25] C. Kessel, D. Campbell, Y. Gribov, G. Saibene, G. Ambrosino, R. Budny, T. Casper, M. Cavinato, H. Fujieda, R. Hawryluk, L. Horton, A. Kavin, R. Kharyrutdinov, F. Koechl, J. Leuer, A. Loarte, P. Lomas, T. Luce, V. Lukash, M. Mattei, I. Nunes, V. Parail, A. Polevoi, A. Portone, R. Sartori, A. Sips, P. Thomas, A. Welander, J. Wesley, Nuclear Fusion 49 (2009) 085034. URL: http://stacks. iop.org/0029-5515/49/i=8/a=085034.

[26] S. H. Kim, J. F. Artaud, V. Basiuk, V. Dokuka, R. R. Khayrutdinov, J. B. Lister, V. E. Lukash, Plasma Physics and Controlled Fusion 51 (2009) 105007. URL: http://stacks.iop.org/0741-3335/51/i=10/a=105007.

[27] S. E. Segre, Plasma Phys. Control Fusion 41 (1999) R57. URL: http: //stacks. iop.org/0741-3335/41/i=2/a=001.

[28] R. Imazawa, Y. Kawano, Y. Kusama, Nuclear Fusion 51 (2011) 113022.

[29] J. Chrzanowski, Y. Kravtsov, Nukleonika 58 (2013) 281-285.

[30] J. Nocedal, S. J. Wright, Numerical optimization, Springer Series in Operations Research and Financial Engineering, second ed., Springer, New York, 2006 .

[31] J. P. Freidberg, Ideal Magnetohydrodynamics, Plenum US, 1987.

[32] J. Wesson, Tokamaks, The International Series of Monographs in Physics, Oxford University Press, 2004.

[33] J. P. Goedbloed, S. Poedts, Principles of magnetohydrodynamics: with applications to laboratory and astrophysical plasmas, Cambridge university press, 2004. 
[34] S. Jardin, Computational methods in plasma physics, Boca Raton, FL : CRC Press/Taylor \& Francis, 2010.

[35] H. Heumann, J. Blum, C. Boulbe, B. Faugeras, G. Selig, J.-M. Ané, S. Brémond, V. Grangirard, P. Hertout, E. Nardon, J. Plasma Physics (2015). doi http://dx.doi.org/10.1017/S0022377814001251

[36] R. Albanese, J. Blum, O. Barbieri, in: EPS. 8th Europhysics Conference on Computing in Plasma Physics (1986), pp. 41-44.

[37] G. Gatica, G. Hsiao, J. Math. Anal. Appl. 189 (1995) 442-461. URL: http: //dx.doi.org/10.1006/jmaa.1995.1029, doi 10.1006/jmaa.1995.1029.

[38] V. Grandgirard, Modélisation de l'équilibre d'un plasma de tokamak, Ph.D. thesis, Université de Franche-Comté, 1999.

[39] J. Blum, J. Le Foll, B. Thooris, Computer Physics Communications 24 (1981) 235 - 254. URL: http://www.sciencedirect.com/science/ article/pii/0010465581901491. doi 10.1016/0010-4655(81)90149-1.

[40] M. Hinze, R. Pinnau, M. Ulbrich, S. Ulbrich, Optimization with PDE constraints, volume 23 of Mathematical Modelling: Theory and Applications, Springer, New York, 2009.

[41] ITM, Integrated Tokamak Modelling, http://portal.efda-itm.eu/, 2013.

[42] G. Falchetto, D. Coster, R. Coelho, B. Scott, L. Figini, D. Kalupin, E. Nardon, S. Nowak, L. Alves, J. Artaud, V. Basiuk, J. P. Bizarro, C. Boulbe, A. Dinklage, D. Farina, B. Faugeras, J. Ferreira, A. Figueiredo, P. Huynh, F. Imbeaux, I. Ivanova-Stanik, T. Jonsson, H.-J. Klingshirn, C. Konz, A. Kus, N. Marushchenko, G. Pereverzev, M. Owsiak, E. Poli, Y. Peysson, R. Reimer, J. Signoret, O. Sauter, R. Stankiewicz, P. Strand, I. Voitsekhovitch, E. Westerhof, T. Zok, W. Zwingmann, ITM-TF Contributors, the ASDEX Upgrade Team, JET-EFDA Contributors, Nuclear Fusion 
54 (2014) 043018. URL: http://stacks.iop.org/0029-5515/54/i=4/a= 043018

[43] T. Yamaguchi, Y. Kawano, H. Fujieda, K. Kurihara, M. Sugihara, Y. Kusama, Plasma Phys. Control Fusion 50 (2008) 045004. 